\title{
Geometrical thermodynamics and P-V criticality of charged accelerating AdS black holes
}

\author{
Kh. Jafarzade ${ }^{1,2 *}$, J. Sadeghi ${ }^{1,2 \dagger}$, B. Eslam Panah ${ }^{1,3,2,4 \ddagger}$ and S. H. Hendi ${ }^{5,6 \S}$ \\ 1 Department of Physics, Sciences Faculty, University of Mazandaran, P. O. Box 47415-416, Babolsar, Iran \\ 2 ICRANet-Mazandaran, University of Mazandaran, P. O. Box 47415-416, Babolsar, Iran \\ ${ }^{3}$ Research Institute for Astronomy and Astrophysics of Maragha (RIAAM), P.O. Box 55134-441, Maragha, Iran \\ 4 ICRANet, Piazza della Repubblica 10, I-65122 Pescara, Italy \\ 5 Department of Physics, School of Science, Shiraz University, Shiraz 71454, Iran \\ ${ }^{6}$ Biruni Observatory, School of Science, Shiraz University, Shiraz 71454, Iran
}

\begin{abstract}
The unusual asymptotic structure of the accelerating black holes led to ambiguity in their geometric characteristics and thermodynamic behavior. Motivated by the interesting properties of such black holes and the significant role of electric charge and string tension on their structure, we study the thermodynamic behavior of these black holes by two methods and examine the changes of free parameters on the thermal behavior of the black holes. First, we investigate phase transition and thermal stability of the system through the use of heat capacity in the non-extended phase space. We examine the effects of electric charge, string tension and the cosmological constant on the phase transition and stability of the system. We also find that to have a phase transition, we have to apply some constraints on the free parameters. Then, we employ the geometrical thermodynamic (GT) method to study phase transition and compare the obtained results with those of the heat capacity. Next, we work in the extended phase space by considering the cosmological constant as a dynamical pressure and evaluate the existence of van der Waals like phase transition. We obtain critical quantities and study the effective role of electric charge and string tension on these quantities. Finally, we make use of the GT method in the extended phase space and find that the results of the GT method, heat capacity and $P-V$ diagram lead to a consistent conclusion.
\end{abstract}

\section{INTRODUCTION}

Black hole thermodynamics is one of the exciting and challenging subjects in theoretical physics. The historical study of the black hole as a thermodynamic system backs to the pioneering works of Bardeen, Carter, Hawking [1, 2] and Bekenstein [3, 4]. They clarified the laws of black hole mechanics and showed that these laws are corresponding to ordinary thermodynamics by appropriate identification of the related quantities, such as temperature, entropy, energy and so on. The investigation of black hole thermodynamics in anti-de Sitter (AdS) spacetime has been aroused the attention of researchers. Studying thermodynamic properties of AdS black holes is preliminary steps of investigating the quantum nature of gravity, one of the most important theoretical subjects in physical communities. Besides, according to the AdS/CFT correspondence, the gravity side on asymptotically AdS spacetime is equivalent to the conformal field theory in one fewer dimension [5 9 ].

Among the various topics in black hole thermodynamics, the phase transition is of particular importance. At first, Hawking and Page [10] demonstrated the existence of a certain phase transition (so-called Hawking-Page) between thermal AdS and Schwarzschild-AdS black hole which corresponds to the confinement/deconfinement phase transition in the dual strongly coupled gauge theory. Afterward, some efforts were conducted in the context of phase transition for more complicated backgrounds 11, 12. Considering the phase transition of charged AdS black holes showed that critical behavior of Reissner-Nordström-AdS black holes is superficially analogous to the van der Waals liquid-gas phase transition [13, 14]. It has been proposed that such similarity will be more precise by considering the cosmological constant as a dynamical pressure in the extended phase space [15, 16]. The consideration of cosmological constant as a thermodynamical pressure and its conjugate quantity as a thermodynamical volume gives us a better insight into understanding van der Waals like behavior, the first and second order phase transitions of black holes. In this regard, some efforts have been made in the context of $P-V$ criticality of black holes in modified theories of gravitation, such as Gauss-Bonnet gravity [17 20], dilaton gravity [21, 22], Lovelock gravity [23 25], Horava-Lifshitz gravity 2630], massive gravity [31 40], $F(R)$ gravity [41, 42], gravity's rainbow [43, 44], and massive gravity's rainbow [45]. Moreover, the effects of linear and nonlinear electrodynamics on the $P-V$ criticality of black holes have been studied

\footnotetext{
* email address: khadije.jafarzade@gmail.com

$\dagger$ email address: pouriya@ipm.ir

$\ddagger$ email address: eslampanah@umz.ac.ir (corresponding author)

$\S$ email address: hendi@shirazu.ac.ir
} 
before [46 [56]. Indeed, the variety of gravitating systems enriches our knowledge of the phase structure of different black holes.

Among different methods to investigate phase transition, studying the heat capacity of a thermodynamical system is more common. Using the heat capacity, one can investigate two distinctive points as bound and phase transition points. The bound point is where the sign of temperature is changed. Since the positive (negative) temperature is representing a physical (non-physical) solution, this point is a limitation point, distinguishing the physical black hole from the non-physical one. The existence of a direct relationship between the heat capacity and the temperature helps us to find these two quantities usually share the same roots. So, one concludes that the bound point is where the numerator of heat capacity (temperature) vanishes. On the other hand, the phase transition point is related to divergency of the heat capacity. Considering the heat capacity also provides a mechanism to study thermal stability of the system. Indeed, the positivity of heat capacity guarantees thermal stability of the system, while its negativity indicates that the system under consideration is in an unstable state [57 61]. Since investigating thermal stability/instability of the system is possible through the heat capacity, we study the behavior of this thermodynamic quantity for charged accelerating black holes and inspect the effects of acceleration (string tension) and electric charge in order to have a stable system. We also obtain a relation between the free parameters of the model for having phase transition. We explore this special condition in the non-extended phase space via studying the heat capacity.

Another formalism for the investigation of black hole phase transition is geometrical thermodynamics (GTs). This formalism is based on the construction of a thermodynamical metric by using thermodynamical potential (internal energy or entropy) and its derivative with respect to the extensive parameters of system. In GTs, a metric is introduced on the equilibrium thermodynamical phase space. In fact, a sort of Riemannian metric is defined as the Hessian of the thermodynamical potential, where the derivatives are taken with respect to the extensive thermodynamic variables. Depending on the choice of the thermodynamical potential, the components of the phase space differ in their structures (this is because each thermodynamical quantity has its specific extensive parameters). The divergencies of Ricci scalar in the constructed metric provide information related to the phase transition. Studying GTs was first began by Weinhold [62, 63]. He introduced a metric on the space of equilibrium states where its components are given as the second derivatives of internal energy with respect to entropy and other extensive quantities. Then, Ruppeiner introduced another metric which is defined as the negative second derivatives of entropy with respect to the internal energy and other extensive quantities [64, 65]. It is shown that there is a conformally relationship between Weinhold and Ruppeiner metrics where the conformal factor is the inverse of temperature [66]. These metrics encounter with some problems. Indeed, the obtained curvature scalars from Weinhold and Ruppeiner metrics are known to diverge at the critical points of usual systems which indicated by a singularity in the specific heat. But for black holes, there are some contradictions. For example, the curvature scalar of the Weinhold metric is singular for ReissnerNordström black hole, which its singularity is not consistent with the singularity of specific heat. The mentioned problem comes from the fact that both the Weinhold and Ruppeiner metrics are not Legendre invariant and so they are not suitable to describe thermodynamic properties of various black holes [67, 68]. In this regard, Quevedo proposed a new type of thermodynamical metric which was invariant under Legendre transformation [67, 68]. The importance of Legendre invariance lies in the thermodynamics itself, meaning that once a representation is chosen to describe a system, its Legendre transform contains the same information as the original representation. In fact, Legendre invariance is an essential ingredient of a geometric construction [69]. Another thermodynamical metric is also introduced by Mansoori et al. [70, 71]. But, these thermodynamic metrics had some shortcomings. Hence, Hendi et al. proposed a new metric that eliminated the problems of previous thermodynamical metrics (see the Refs. [72 76], for more details on various black holes). It is worthwhile to mention that this new thermodynamical metric is defined the same as Quevedo's metric with a different functional form of the conformal factor. Recently, two new metrics were introduced by geometric interpretation of criticality conditions where the divergency of the Ricci scalar of these metrics is representing critical point $77,[78$. It is worth mentioning that the basic motivation of GTs is to give an independent picture regarding thermodynamical aspects of systems. In addition, GTs provides information regarding bound points, phase transitions, their types and stability conditions. Furthermore, it can give microscopic information about a thermodynamic system. In other words, by using the sign of thermodynamical Ricci scalar, one can study the kind of intermolecular interaction along the transition curve. Positivity (negativity) of $R$ refers to the dominance of repulsive (attractive) interaction in the thermodynamic system, whereas $R=0$ indicates that there is no interaction in such a system 64]. Since Ruppeiner geometry is obtained from the thermodynamic fluctuation theory [65], it is usual to employ such geometry to probe the microstructure of a thermodynamic system. Pineda et al. have recently displayed a direct connection between Legendre invariant metrics and fluctuation theory [79]. In Ref. [69], Quevedo and Tapias used the formalism of GTs to describe chemical reactions in the context of equilibrium thermodynamics and showed that the curvature of the equilibrium manifold with a Legendre invariant metric reflects the thermodynamical interaction. They conducted their study in the context of an ideal gas and a van der Waals fluid. In this paper, we employ the mentioned thermodynamic metrics and show which one can provide a suitable picture of phase transition of charged accelerating black holes in both extended and non-extended phase space. According 
to the critical conditions, we also illustrate that the introduced metrics are a suitable candidate for only spherical symmetric black holes, not for accelerating ones. Besides, we employ the GT idea to investigate the micro-structure of charged accelerating black holes that have van der Waals like behavior and show that the microscopic properties of such black holes are similar to those of van der Waals fluid.

In recent years, the accelerating black holes attract much attention 80 93]. These black holes are described by the C-metric [94 97], interpreting as two uniformly accelerating black holes. One of the main properties of this metric is the existence of a string-like singularity along one polar axis attached to the black hole. This conical singularity can be imagined as a cosmic string with a tension providing the force driving the acceleration. It is noteworthy that these conical singularities can be eliminated after imposing appropriate restrictions on physical parameters and then embedding the solutions into $D=11$ supergravity. Besides, an interesting feature of the accelerating black holes is their uncommon asymptotic behavior where the curvature of background is not constant at spatial infinity. Moreover, depending on the range of the parameters, an acceleration horizon can be appeared, causing a complicated structure. The existence of such a horizon raises the problem of thermodynamic equilibrium. Considering a negative cosmological constant, one can eliminate this problem [80]. The mentioned black hole is called a slowly accelerating and is displaced from the center of AdS spacetime via a cosmic string ending on the black hole horizon [98]. The thermodynamics of such black holes was investigated by Appels et al in Ref. [80]. They showed that the first law of these black holes can be expressed in the standard form under the satisfaction of two certain conditions. Another interesting feature is related to the fact that the circular orbits of the photons deviate from the equatorial plane and the property of the black hole shadow changes due to the acceleration. Indeed, the latitude of the circular orbit increases by increasing the acceleration [99].

Within general relativity (GR), the C-metric has been used to investigate radiation at infinity [100, 101]. However, the application of C-metric is not limited to GR. It can be used to describe the production of black hole pairs in strong background fields [102], the construction of the black ring solution in five dimensions [103], the generation of black holes in an electric or magnetic field and the splitting of cosmic strings [104, 105]. Since the oppositely accelerating black holes are causally disconnected and the metric can be expressed in appropriate coordinates to cover just one of the "moving" black holes, C-metric can be regarded as a reasonable candidate to describe boosted black holes [106]. Besides, this metric has been generalized to include rotation, cosmological constant and a Newman-Unti-Tamburino (NUT) parameter [95]. The gravitational lensing [99, 107, 108], quasinormal modes [109], holographic complexity 110], stability of the Cauchy horizon [111], gravitational entropy [112], spinning spindles [113] and thermodynamics 114 117] of accelerating black holes have been investigated before. Moreover, the effects of $f(R)$ gravity [118 120] and NUT parameter [121] on the properties of accelerating black holes have been reported in the litrature.

It was shown that depending on the gravity under consideration and employed matter fields, the critical behavior of the system may be modified and some conditions regarding the existence/absence of van der Waals like behavior may appear. So, it would be interesting to investigate how the acceleration of the black hole affects its phase transition. In the present paper, we also study the van der Waals like behavior, critical properties and conditions for observing the critical behavior of these black holes.

We organize this paper as follows: in Sec. III we briefly review the charged accelerating AdS black holes. In Sec. [II] we investigate the phase transition of these black holes by using the heat capacity and GT method in non-extended phase space and study thermal stability conditions. Section IV devoted to employ the analogy between the cosmological constant and thermodynamical pressure and extend phase space. We investigate van der Waals like phase transition and obtain the critical point by two different methods and show that their results are identical. Then, we study the critical behavior of the system via heat capacity and GT method and indicate that these approaches lead to the same results. In the last section, we present our conclusions.

\section{CHARGED ACCELERATING ADS BLACK HOLES}

In this section, we review some basic properties of the charged accelerating AdS black holes. The metric governing the charged accelerating black holes is [80]

$$
d s^{2}=\frac{1}{\Omega^{2}}\left[f(r) d t^{2}-\frac{d r^{2}}{f(r)}-r^{2}\left(\frac{d \theta^{2}}{g(\theta)}+g(\theta) \sin ^{2} \theta \frac{d \phi^{2}}{K^{2}}\right)\right]
$$

where

$$
\begin{aligned}
& f(r)=\left(1-A^{2} r^{2}\right)\left(1-\frac{2 m}{r}+\frac{e^{2}}{r^{2}}\right)+\frac{r^{2}}{\ell^{2}}, \\
& g(\theta)=1+2 m A \cos \theta+e^{2} A^{2} \cos ^{2} \theta,
\end{aligned}
$$


also $\Omega$ is conformal factor which is given by

$$
\Omega=1+A r \cos \theta
$$

This factor determines the conformal infinity or boundary of the AdS spacetime. The parameters $A(A>0)$ and $\ell=\sqrt{\frac{-3}{\Lambda}}(\Lambda$ is cosmological constant) are the acceleration parameter and AdS radius, respectively. Also, the parameters $m$ and $e$ are related to the mass and electric charge of the black hole as [86, 122, 123]

$$
\begin{aligned}
& M=\left[1-\left(1+A^{2} e^{2}\right) A^{2} \ell^{2}\right] \frac{m}{K \alpha}, \\
& Q=\frac{1}{4 \pi} \int_{\Omega=0} * F=\frac{e}{K},
\end{aligned}
$$

where the parameter $K$ encodes information about the conical deficit on the south and north poles, so that $\phi \in[0,2 \pi]$. In addition, the parameter $\alpha$ is a normalization factor, re-scaling the time coordinate, which is necessary to have a welldefined thermodynamic behavior [83, 86, 87, 124]. Considering the normalization factor, the proper time coordinate of an asymptotic observer is $\tau=\alpha t$, in which $\alpha=\sqrt{\left(1+e^{2} A^{2}\right)\left(1-A^{2} \ell^{2}\left(1+e^{2} A^{2}\right)\right)}$ (more details are given in appendix A). The electromagnetic field two-form $F$ is related to gauge potential one form $B$ as [87]

$$
F=d B, \quad \& \quad B=-\frac{e}{\alpha}\left(\frac{1}{r}-\frac{1}{r_{+}}\right) d t .
$$

It is to be noted that the conical singularity in Eq. (1) is removed by adjusting $m=e=0$, the resultant spacetime is a pure AdS metric in Rindler-type coordinates. We will introduce a proper coordinate transformation in appendix B to remove this problem.

Taking a close look at the angular part of metric and the behavior of $g(\theta)$ at poles $\theta_{+}=0(\cos 0=1)$ and $\theta_{-}=\pi$ $(\cos \pi=-1)$, one can find the presence of cosmic string. The regularity of metric at a pole leads to

$$
K_{ \pm}=g\left(\theta_{ \pm}\right)=1 \pm 2 m A+e^{2} A^{2}
$$

As it is seen in Eq. (6), for $m A \neq 0$, one cannot fix the parameter of $K$ and therefore, there exists irregularity at both poles. The irregularity at an axis causes the conical singularity. Thus, $K$ is chosen to regularize one pole, leaving either a conical deficit or a conical excess along the other pole. The conical deficits on the north pole $\left(\theta_{+}=0\right)$ and the south pole $\left(\theta_{-}=\pi\right)$ are given by

$$
\delta_{ \pm}=2 \pi\left(1-\frac{g\left(\theta_{ \pm}\right)}{K}\right)
$$

which corresponding to a cosmic string with tension [83, 93]

$$
\mu_{ \pm}=\frac{\delta_{ \pm}}{8 \pi}=\frac{1}{4}-\frac{1 \pm 2 m A+e^{2} A^{2}}{4 K} .
$$

By setting $K=K_{+}=1+2 m A+e^{2} A^{2}$ (i. e., $\mu_{+}=0$ ), one can remove the conical singularity on the north pole $(\theta=0)$. So, there is a conical deficit on the south pole with the string tension $\mu=\mu_{-}=\frac{m A}{K}$. The $m A$-term should be restricted to $m A<\frac{1}{2}$ to preserve the metric signature [83]. For simplicity, we consider $\mathcal{B}=m A$ as a constant parameter throughout this paper.

In order to have a well-defined metric (1), corresponding physically to a slowly accelerated black hole in the bulk, certain conditions should be imposed in the range of different parameters as follows:

I) The function $g(\theta)$ should be a positive definite function for $\theta$ in $[0, \pi]$.

II) Due to the requirement of slow acceleration, the function $f(r)$ should has no root on the boundary $\left(f\left(-\frac{1}{A \cos \theta}\right)>0\right)$.

III) The spacetime has to admit a black hole in the bulk.

We study the behavior of metric function in Fig. 1. One can observe three different cases. a) Two real roots which are Cauchy and event horizons. b) One real root (extreme horizon). c) Absence of real root (naked singularity). Fig. 1. shows regions in which the metric function has one or two real roots.

Our analysis shows that in order to have black hole solutions, one has to consider the small values for the electric charge $(Q)$ and the large values for string tension $(\mu)$, mass $(M)$. 

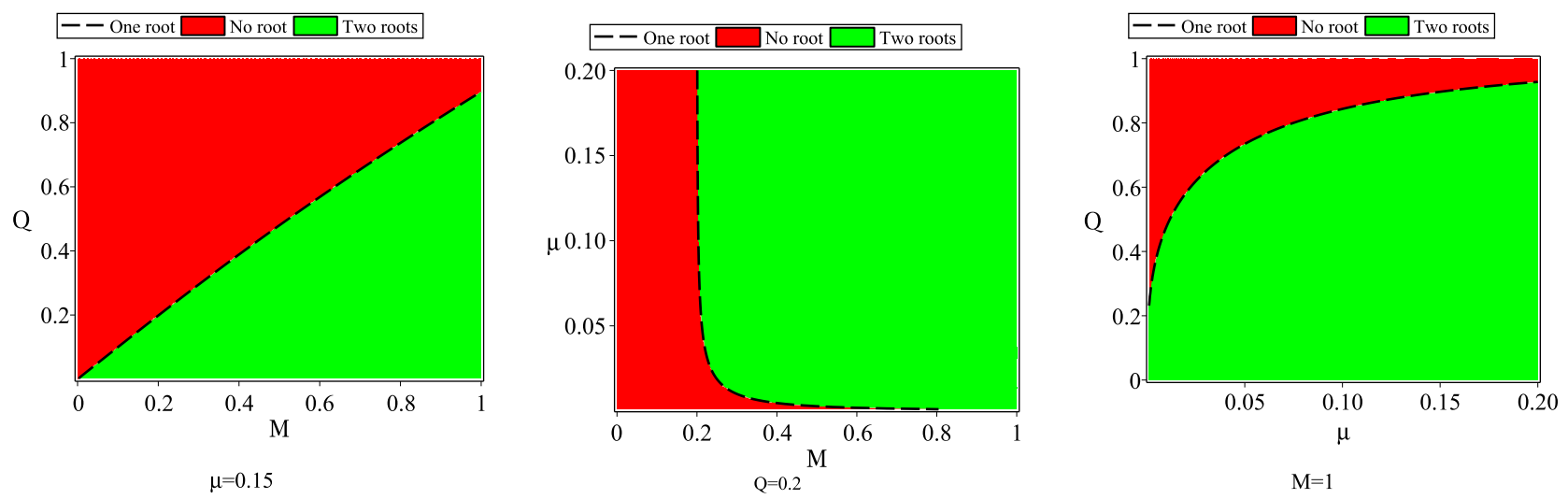

FIG. 1: Variation of $f(r)$ as a function of different parameters for $\mathcal{B}=0.2, \beta=0.04, \ell=2$ and $A=0.02$.

\section{THERMODYNAMICAL STRUCTURE IN NON-EXTENDED PHASE SPACE}

In this section, we study phase transition and thermal stability of the charged accelerating AdS black holes in the context of the canonical ensemble by calculating the heat capacity. We also investigate the effects of electric charge, the AdS radius (or the cosmological constant) and string tension on phase transition and stability of the system and show that a certain relation between these parameters should be satisfied in order to have phase transition. Then, we employ thermodynamical metrics to study phase transition and compare obtained results with those of heat capacity.

\section{A. Temperature and (non)physical black hole solutions}

In order to study the physical black hole solutions, we have to evaluate the behavior of temperature. According to the pioneering work of Hawking, the temperature of black holes is related to the surface gravity $\kappa$ by the relation $T=\frac{\kappa}{2 \pi}$ [2]. Replacing the geometrical mass $m$ which is obtained by evaluating the metric function on horizon $\left(f\left(r=r_{+}\right)=0\right)$ as 125 ]

$$
m=\frac{\left(r_{+}^{2}+e^{2}\right)\left(1-A^{2} r_{+}^{2}\right)+\frac{r_{+}^{4}}{\ell^{2}}}{2 r_{+}\left(1-A^{2} r_{+}^{2}\right)}
$$

in the temperature relation, one can find Hawking temperature as

$$
T=\frac{f^{\prime}\left(r_{+}\right)}{4 \pi \alpha}=\frac{3 r_{+}^{2}+\ell^{2}\left(1-\frac{Q^{2} \mathcal{B}^{2}}{\mu^{2} r_{+}^{2}}\right)-\beta^{2} r_{+}^{2}\left(\ell^{-2} r_{+}^{2}+\left(2-A^{2} r_{+}^{2}\right)\left(1-\frac{Q^{2} \mathcal{B}^{2}}{\mu^{2} r_{+}^{2}}\right)\right)}{4 \pi \alpha r_{+}\left(\ell^{2}-\beta^{2} r_{+}^{2}\right)}
$$

where $\mathcal{B}=m A$ and $\beta=A \ell$, are considered as fixed parameters throughout the paper. We should note that to preserve the metric signature and remove the acceleration horizon, we consider, respectively, $2 \mathcal{B}<1$ and $\beta<1$ [83]. According to the numerator of temperature (Eq. (10)), one can find that it has at least one root. More investigations confirm that there are three roots for the temperature which only real one is

$$
\left.r_{+}\right|_{T=0}=\sqrt{2 \sqrt{\frac{-\rho}{3}} \sin \left(\frac{1}{3} \sin ^{-1}\left(\frac{3 \sqrt{3} g}{2(\sqrt{-\rho})^{3}}\right)\right)+\frac{\left(3-2 \beta^{2}\right)}{3 A^{2}}},
$$

where

$$
\begin{aligned}
& \rho=-\frac{1}{3 A^{4}}\left[9\left(1-\beta^{2}\right)+2 \beta^{4}\left(2+\frac{3 Q^{2} \mathcal{B}^{2}}{\mu^{2} \ell^{2}}\right)\right] \\
& g=-\frac{1}{27 A^{6}}\left[54-81 \beta^{2}+27 \beta^{4}\left(2+\frac{Q^{2} \mathcal{B}^{2}}{\mu^{2} \ell^{2}}\right)+4 \beta^{6}\left(4+\frac{9 Q^{2} \mathcal{B}^{2}}{\mu^{2} \ell^{2}}\right)\right] .
\end{aligned}
$$


Taking a look at Eq. (10), one can see that the temperature diverges at $A r_{+}=1$. To investigate the effects of black hole parameters on the temperature, we have plotted Fig. 2.

Analyzing the temperature for small and large values of the horizon radius, gives us interesting information regarding these black holes. Using the series expansion of $T$ for vanishing horizon radius (or very small charged accelerating black holes) results into

$$
\lim _{r_{+} \longrightarrow 0} T \propto-\frac{Q^{2} \mathcal{B}^{2}}{4 \pi \alpha \mu^{2} r_{+}^{3}}+\frac{1}{4 \pi \alpha r_{+}}\left(1+\frac{A^{2} Q^{2} \mathcal{B}^{2}}{\mu^{2}}\right)+O\left(r_{+}\right),
$$

which confirms the following important points:

I) The only quantity which does not have direct effect on the high energy limit of the temperature is the AdS radius.

II) The dominant term in the high energy limit of the temperature which includes the electric charge and string tension is always negative. It means that the temperature diverges at $r_{+} \longrightarrow 0$ for non-vanishing $B, Q$ and $\mu$. Since negative temperature corresponds to a non-physical solution, there is no physical solution for a very small charged accelerating black hole. Indeed, by decreasing the event horizon radius, one can find that the temperature vanishes before it negatively diverges.

III) In the absence of electric charge, the temperature is always positive, indicating that a very small uncharged accelerating black hole has a physical solution, unlike its charged counterpart.

The asymptotic behavior of the temperature is given by

$$
\lim _{r_{+} \longrightarrow \infty} T \propto \frac{\left(1-\beta^{2}\right) r_{+}}{4 \pi \alpha \ell^{2}}+O\left(\frac{1}{r_{+}}\right),
$$

which shows that for large black holes, the temperature is governed by the AdS radius. As it was mentioned, an accelerating black hole has an acceleration horizon. To resolve such a problem, one should consider $\beta<1$ [83]. So, existence of such a constraint ensures physical solution for large black holes.

Now, we focus on the behavior of the temperature and the effects of electric charge and string tension on it. As one can see from Fig. 2. the temperature is zero for certain values of the horizon radius. At this point $\left(r_{+}=r_{0}\right)$, the sign of temperature changes from negative to positive. Where for $\left(r_{+}<r_{0}\right)$, the temperature is always negative which is representing a non-physical solution. But for $r_{+}>r_{0}$, the temperature is positive and the black hole has a physical solution.

The first parameter that we study its effect on the temperature is the electric charge $(Q)$. The left panel of Fig. 2. shows that $r_{0}$ increases by increasing $Q$. Therefore, the region of the non-physical solution increases. Here, the interesting issue is that as $Q$ decreases, the temperature acquires one or two extrema. Evidently, in extremum, the denominator of the heat capacity is zero. In other words, the heat capacity diverges at this point. Then, one can say that extrema are the same points in which the black hole undergoes a phase transition. Increasing $Q$, these extrema disappear and only a bound point will be observed. So, by using the temperature diagram, one can study both bound and phase transition points. On the other hand, by employing this diagram, one can achieve a better insight in understanding single phase regions which are small/large black holes. The region $r_{0}<r_{+}<r_{\max }$ is related to small black holes and the region $r_{+}>r_{\min }$ is denoted by large black holes $\left(r_{\max }\right.$ and $r_{\min }$ are the first and second extremum, respectively). Medium black holes are located between these two extrema (see Refs. [60, 126], for more details).

The next parameter which affects the temperature is the string tension. Taking a closer look at the right panel of Fig. 2 one can find that the role of $\mu$ is opposite of that of electric charge. In other words, by increasing $\mu$, the extrema are formed while for its small values, the only bound point is observed. Also, one can see that as the string tension increases the bound point shifts to a smaller horizon radius.

\section{B. Heat capacity and phase transition}

Heat capacity is one of the interesting thermodynamical quantities which describes two important issues of a thermodynamical system as thermal stability and phase transition. The positivity of heat capacity indicates thermal stability of the system while its negativity is representing instability. One can consider two cases for an unstable black hole. It may go to a stable state by a phase transition or it has a non-physical solution which in this case no phase transition takes place. So, we can extract two characteristic points by using the heat capacity: bound and phase transition points. As it was already mentioned [60], the bound point is related to the root of heat capacity. Whereas, the phase transition point(s) is(are) where heat capacity diverges. The heat capacity is given by 

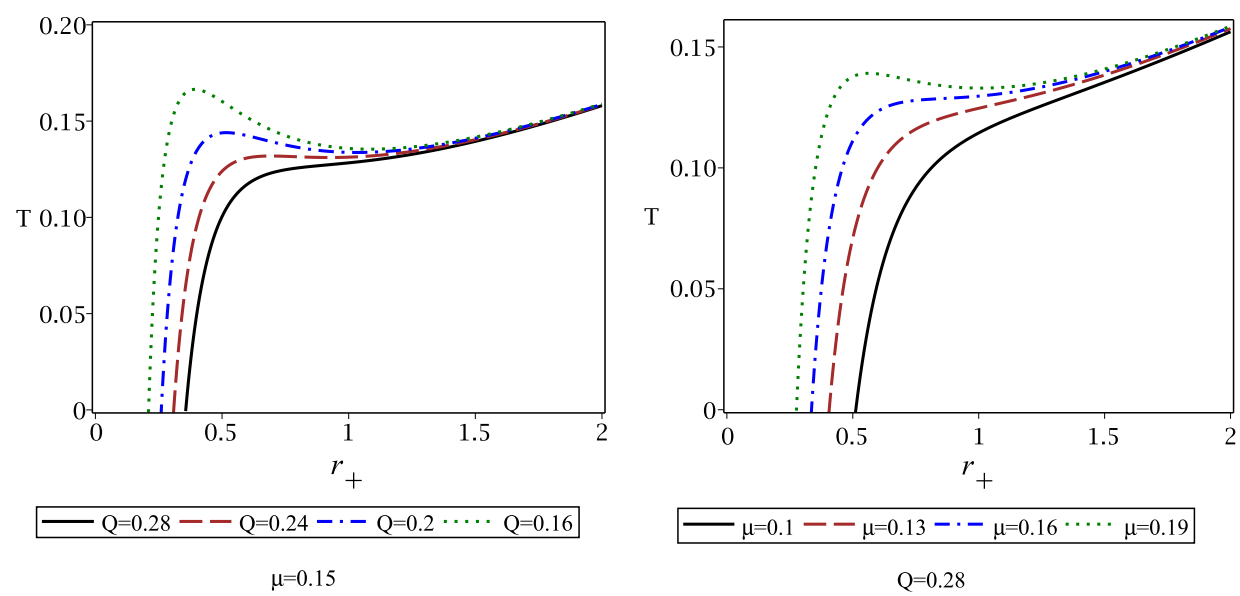

FIG. 2: $T$ versus $r_{+}$for $\mathcal{B}=0.2, \beta=0.04, \ell=2$ and $A=0.02$. Left panel for different values of the electric charge. Right panel for different values of the string tension.

$$
C_{Q, \mu}=T\left(\frac{\partial S}{\partial T}\right)_{Q}=\frac{\left(\frac{\partial M}{\partial S}\right)_{Q}}{\left(\frac{\partial^{2} M}{\partial S^{2}}\right)_{Q}}
$$

The entropy $S$ of the black hole is identified with a quarter of the horizon area as

$$
S=\frac{\mathcal{A}}{4}=\frac{\pi r_{+}^{2}}{K\left(1-A^{2} r_{+}^{2}\right)} .
$$

It is evident that the entropy diverges at $A r_{+}=1$. To avoid divergency and negatively, one should consider $A<\frac{1}{r_{+}}$. As it was pointed out, in the canonical ensemble, the phase transition points are detected as divergence points of the heat capacity. By employing Eqs. (16), (15) and (10), one finds

$$
C_{Q, \mu}=\frac{2 \pi \mu r_{+}^{2}\left(3 r_{+}^{2}\left(1-\frac{A^{2} r_{+}^{2}}{3}\right)+\ell^{2}\left(1-\frac{Q^{2} \mathcal{B}^{2}}{\mu^{2} r_{+}^{2}}\right)\left[1-2 A^{2} r_{+}^{2}\left(1-\frac{A^{2} r_{+}^{2}}{2}\right)\right]\right)}{\left(1-A^{2} r_{+}^{2}\right)\left(3 r_{+}^{2}\left(1+\frac{A^{2} \beta^{2} X}{3 \mu^{2}}\right)+\ell^{2}\left(1-\frac{7 Q^{2} \mathcal{B}^{2}}{\mu^{2} r_{+}^{2}}\right)\left(\beta^{2} r_{+}^{2}-1\right)-\frac{4 \ell^{2} Q^{2} \mathcal{B}^{2}}{\mu^{2} r_{+}^{2}}\right) \mathcal{B}},
$$

where

$$
X=\mu^{2} r_{+}^{2}\left(1-A^{2} r_{+}^{2}\right)\left(1+\frac{Q^{2} \mathcal{B}^{2}}{\mu^{2} r_{+}^{2}}\right)+4 Q^{2} \mathcal{B}^{2}+\frac{\mu^{2} r_{+}^{4}}{\ell^{2}}
$$

To understand the heat capacity in more details, we investigate the its limiting behaviors as

$$
C_{Q, \mu} \Rightarrow\left\{\begin{array}{l}
\lim _{r_{+} \longrightarrow 0} C_{Q, \mu} \propto-\frac{2 \pi \mu r_{+}^{2}}{3 \mathcal{B}}+O\left(r_{+}^{4}\right) \text { small black holes } \\
\lim _{r_{+} \longrightarrow \infty} C_{Q, \mu} \propto \frac{2 \pi \mu}{r_{+}^{2} A^{4} \mathcal{B}}+O\left(\frac{1}{r_{+}^{4}}\right), \text { large black holes }
\end{array} .\right.
$$

For small black holes: as it was already mentioned, there is a physical solution in this limit just in the absence of electric charge (see Eq. 13). But as we see, they are not thermally stable due to the negativity of heat capacity.

For large black holes: the heat capacity is highly governed by the acceleration parameter and string tension and due to the positivity of heat capacity in this limit, these black holes are thermally stable.

To study the behavior of heat capacity and the effects of different parameters on this quantity, we have plotted some diagrams in Figs. 3. and 4. Evidently, the heat capacity has only one root for $Q>0.2502$ at $r_{+}=r_{0}$ which is a bound point (see the up panels of Fig. 3). For $r_{+}<r_{0}$, both temperature and heat capacity are negative and so there is no physical solution. In contrast, for $r_{+}>r_{0}$, thermally stable phase can be observed for such black holes. Up panels of Fig. 3. show that the heat capacity has a root and two divergencies $\left(r_{D i v 1}\right.$ and $r_{D i v 2}$ where $\left.r_{D i v 1}<r_{D i v 2}\right)$ for small 
electric charges. Between root and the smaller divergency, the heat capacity has a positive value and black holes which are placed in this region are in a stable phase. The region between two divergencies is related to medium black holes that due to the negativity of heat capacity, they are thermally unstable. For region after the larger divergency, the heat capacity is positive and large black holes are in a stable phase. As it was pointed out, medium black holes are in an unstable phase and undergo a phase transition.
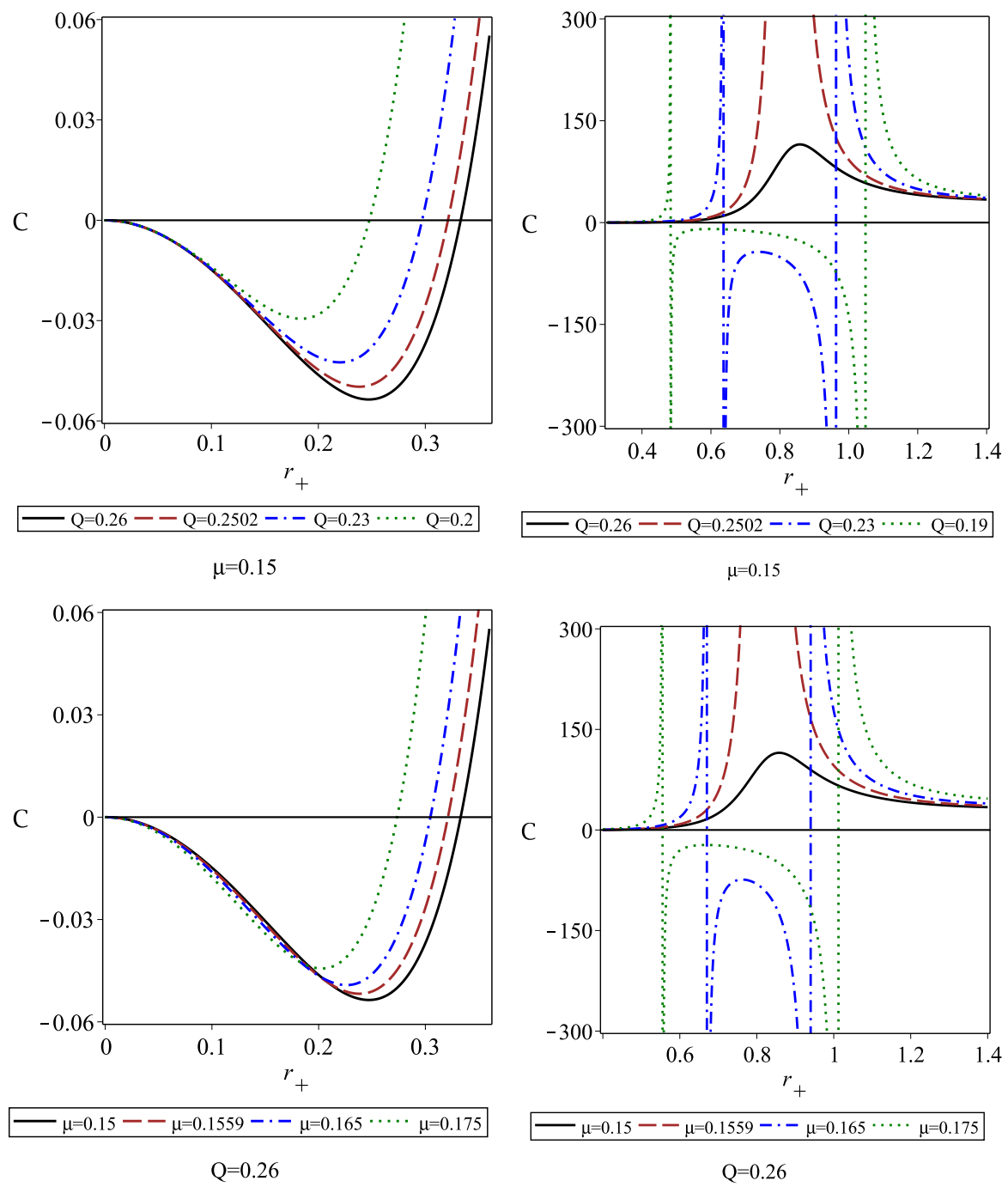

FIG. 3: $C_{Q, \mu}$ versus $r_{+}$for $\mathcal{B}=0.2, \beta=0.04, \ell=2$ and $A=0.02$. Up panels for different values of the electric charge (for different scales). Down panels for different values of the string tension (for different scales).

Now, we would like to study the crucial role of string tension on the phase transition of the system. In the bottom panels of Fig. 3, we have considered $Q$ as a fixed parameter and investigated the behavior of heat capacity for different values of $\mu$. From up panels of Fig. 3, we saw that only charged accelerating black holes with small electric charge enjoy the existence of phase transition. But down panels of Fig. 3, show that such black holes undergo phase transition by increasing the string tension.

As it was mentioned [83], accelerating black holes have well-defined thermodynamics just under satisfaction of the certain condition $A \ell<1$. The cosmological constant which is proportional to AdS radius is representing the natural curvature of the spacetime. In other words, the curvature is an increasing/a decreasing function of $\ell$ [127]. Now, we are interested to investigate how the AdS radius affects the phase transition of the system. In left and middle panels of Fig. 4, we studied the role of $\ell$ on the heat capacity with fixed $\mu$ and $Q$. As we see, black holes with low acceleration (small string tension) and large electric charge can experience phase transition in a high curvature background.

As a result, one can find that charged accelerating black holes with small electric charge undergo phase transition 

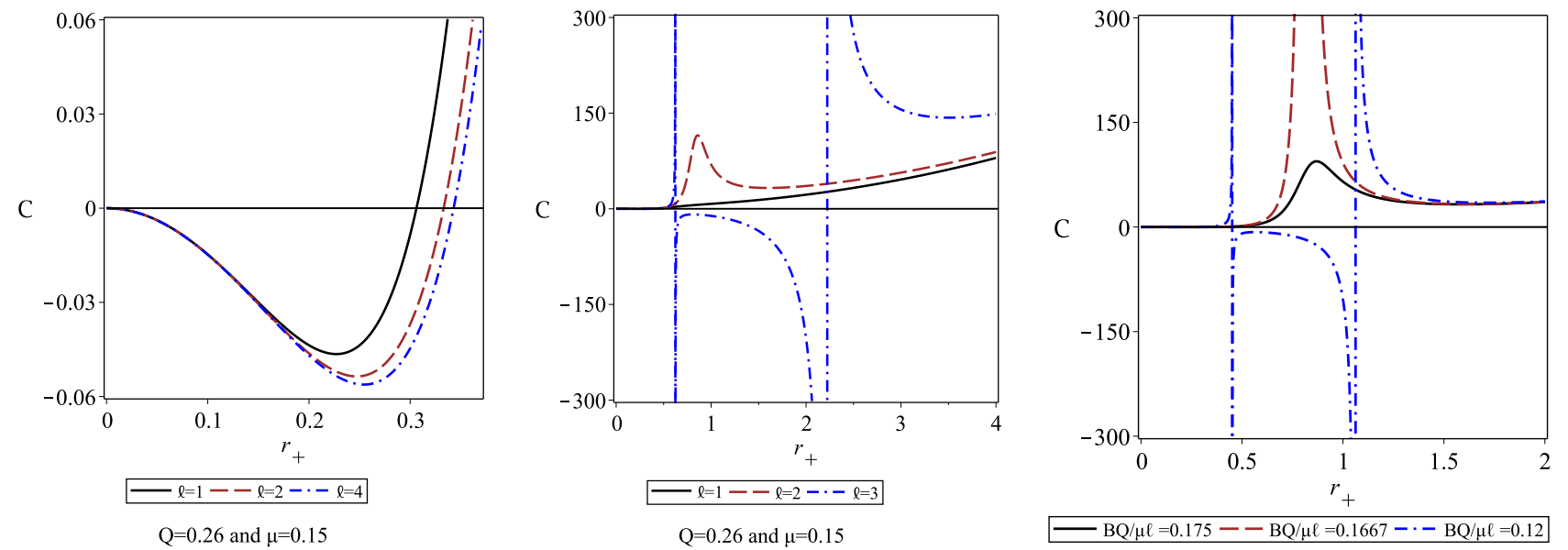

FIG. 4: $C_{Q, \mu}$ versus $r_{+}$for $\mathcal{B}=0.2$. Two left panels for $\beta=0.04, A=0.04$ (continuous line), $A=0.02$ (dashed line), $A=0.01$ (dash-dotted line) and different values of the AdS radius (for different scales). Right panel: the relation between black hole parameters for having phase transition.

easily. But black holes with large electric charge should be pulled by stronger strings or they should be located in a higher curvature background for having a phase transition. Therefore, one can find that there is an implicit relationship between the electric charge, string tension and AdS radius for observing a phase transition. According to the right panel of Fig. 4, we find that for the mentioned parameters, the heat capacity diverges for $\frac{Q \mathcal{B}}{\mu \ell}<0.1667$. In contrast, for $\frac{Q \mathcal{B}}{\mu \ell}>0.1667$, the heat capacity has no phase transition point. For the case of $\frac{Q \mathcal{B}}{\mu \ell}=0.1667$, the heat capacity has only one divergence point where two phases of medium and large black holes are in equilibrium. This indicates that such a condition $\left(\frac{Q \mathcal{B}}{\mu \ell}<0.166\right)$ should be satisfied to have phase transition for charged accelerating black holes.

Now, we focus on the effects of electric charge and string tension on thermal stability of the system. Fig. 3, shows that as the electric charge (string tension) increases (decreases), bound point shifts to the larger horizon radius. So, the physical region is a decreasing (an increasing) function of $Q(\mu)$. Taking a close look at this figure, one can find that as the string tension (electric charge) increases (decreases), $r_{D i v 1}$ decreases whereas $r_{D i v 2}$ increases. So, the unstable region increases by increasing (decreasing) $\mu(Q)$, see also Fig. 5 for more details.
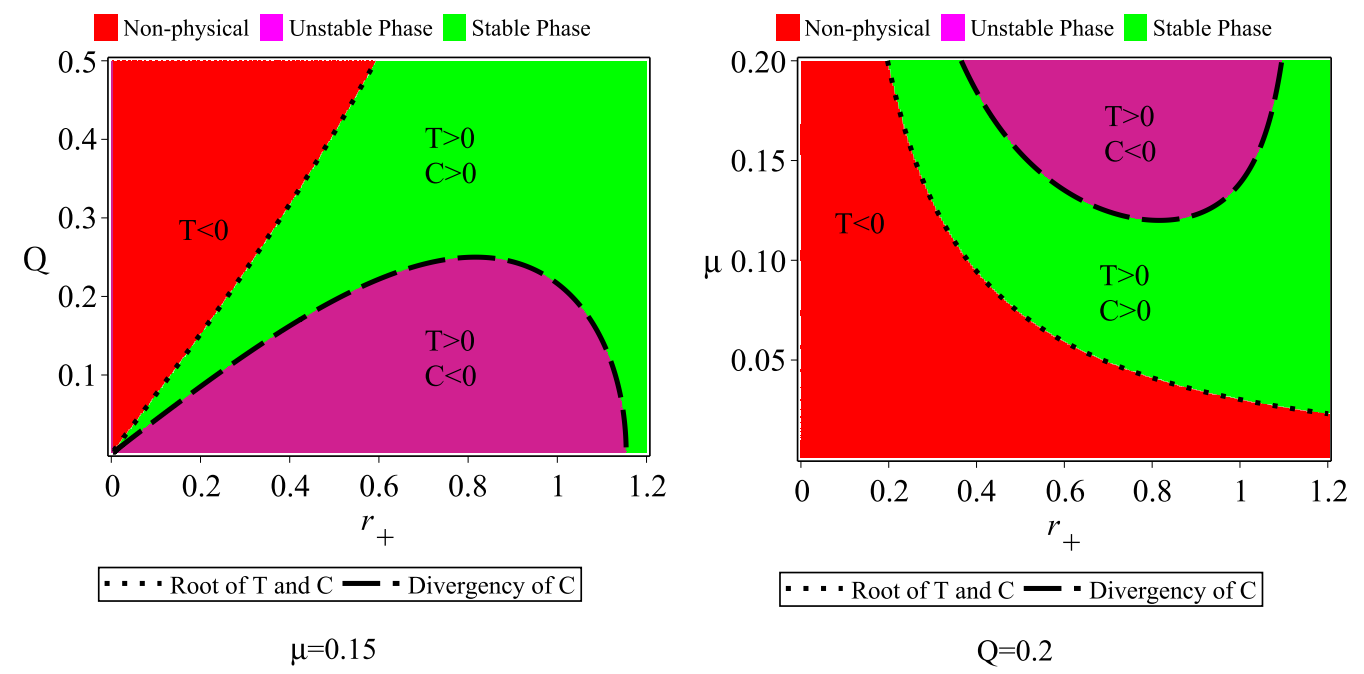

FIG. 5: Thermally stable and/or unstable regions of the black holes for $\mathcal{B}=0.2, \beta=0.04, \ell=2$ and $A=0.02$. 


\section{Geometrical thermodynamics and phase transition}

Another approach to study the thermodynamical behavior of a system is geometrical thermodynamics. In this method, a thermodynamical phase space (metric) is constructed by considering one of the thermodynamical quantities as thermodynamical potential and other quantities as extensive parameters. By calculating the Ricci scalar of such a thermodynamical metric and determining its divergence points, one can obtain phase transition points of the system. It was shown that obtained results are matched with the results of the heat capacity. It means that divergence points of the Ricci scalar and divergence points (and root) of the heat capacity are coincident (see Refs. 72 76], for more details). There are several methods to build thermodynamical metrics known as Weinhold, Ruppeiner, Quevedo and HPEM metrics. Here, we would like to investigate phase transition of the charged accelerating AdS black holes in the non-extended phase space via the GT method. To introduce a suitable metric, we consider the total mass as a thermodynamical potential with the entropy and the electric charge as extensive parameters. The thermodynamical metrics in the context of GTs are given by

$$
d s^{2}=\left\{\begin{array}{cc}
M g_{a b}^{W} d X^{a} d X^{b}, & \text { Weinhold } \\
-T^{-1} M g_{a b}^{W} d X^{a} d X^{b}, & \text { Ruppeiner } \\
\left(S M_{S}+Q M_{Q}\right)\left(-M_{S S} d S^{2}+M_{Q Q} d Q^{2}\right), & \text { Quevedo } \\
S \frac{M_{S}}{M_{Q Q}^{3}}\left(-M_{S S} d S^{2}+M_{Q Q} d Q^{2}\right), & \text { HPEM }
\end{array}\right.
$$

where $M_{k}=\frac{\partial M}{\partial k}$ and $M_{k j}=\frac{\partial^{2} M}{\partial k \partial j}$. Since we are looking for the divergence points of the Ricci scalar and due to the fact that its numerator is a smooth finite function, we focus on its denominator. The denominators of the Ricci scalars are

$$
\operatorname{denom}(\mathcal{R})=\left\{\begin{array}{cc}
\left(M_{S S} M_{Q Q}-M_{S Q}^{2}\right)^{2} M^{2}, & \text { Weinhold } \\
\left(M_{S S} M_{Q Q}-M_{S Q}^{2}\right)^{2} T M^{2}, & \text { Ruppeiner } \\
M_{S S}^{2} M_{Q Q}^{2}\left(S M_{S}+Q M_{Q}\right)^{3}, & \text { Quevedo } \\
S^{3} M_{S}^{3} M_{S S}^{2}, & \text { HPEM }
\end{array} .\right.
$$

Taking Eq. (20) into account, we are in a position to find that among the mentioned metrics, which one is an efficient tool to describe phase transitions of the charged accelerating black holes. To have a proper geometrical approach for studying phase transitions, the thermodynamic Ricci scalar should diverge at points we will mention: bound and phase transition points. Regarding Eq. (20), it is evident that only in a special case $M_{S Q}=0$ and nonzero $M_{Q Q}$, the divergence points of the heat capacity coincide with divergencies of the Weinhold and Ruppeiner Ricci scalars. Due to the presence of the temperature in the denominator of Ruppeiner's Ricci scalar, this metric can describe a bound point, while Weinhold's metric just under the satisfaction of condition $M_{S S} M_{Q Q}-M_{S Q}^{2}=M_{S}$, is able to describe this point. For the case of $M_{S S}=\frac{M_{S Q}^{2}}{M_{Q Q}}$, there are extra divergencies for $R_{W}$ (Weinhold's Ricci scalar) and $R_{R}$ (Ruppeiner's Ricci scalar) which are not related to any phase transition of the heat capacity. Regarding Quevedo's metric, the existence of $M_{S S}$ in the denominator of Ricci scalar guarantees that the divergencies of the heat capacity and Quevedo's Ricci scalar coincide. However, considering the presence of $M_{Q Q}^{2}$, one may find an extra singular point for vanishing $M_{Q Q}$. It is worthwhile to mention that another divergence point may be appeared from zero of $S M_{S}+Q M_{Q}=0$ as well. Regarding the bound point, as we see from the denominator of $R_{Q}$ (Quevedo's Ricci scalar), coincidence with this point takes place only for vanishing $M_{Q}$ (which is in general a nonzero function). We continue our investigation by employing HPEM's metric. Due to the structure of denominator of $R_{H}$ (HPEM's Ricci scalar), the divergence points and root of the heat capacity coincide with divergencies of the HPEM's Ricci scalar. So, this metric provides a successful mechanism for investigating bound and phase transition points of such black holes. In order to have a more precise picture, we have plotted some diagrams in Figs. 6] and 7 which confirm our analysis. A remarkable issue regarding the HPEM's metric is that, in addition to describe bound and phase transition points, one can distinguish these points from each other. It means that the behavior of HPEM's Ricci scalar differs around root and divergencies of $C_{Q, \mu}$ (compare the left panels of Fig. 7 with its right panels). Considering the root of heat capacity, the sign of Ricci scalar near divergence point changes from $-\infty$ to $+\infty$. Whereas, for the case of divergencies of heat capacity the sign of Ricci scalar does not change. So, one can recognize bound point and phase transition points by studying the behavior of the HPEM's Ricci scalar around its divergencies. 


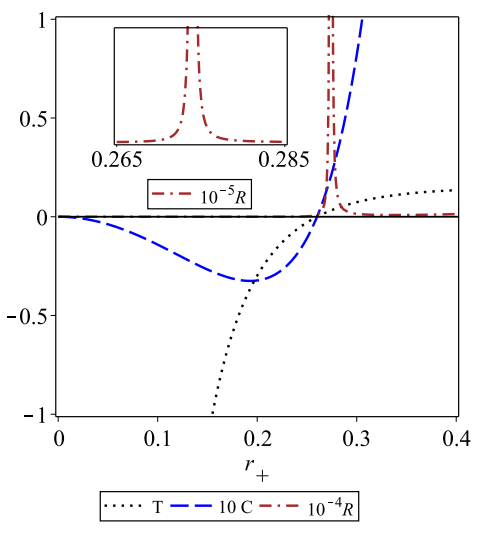

(a)

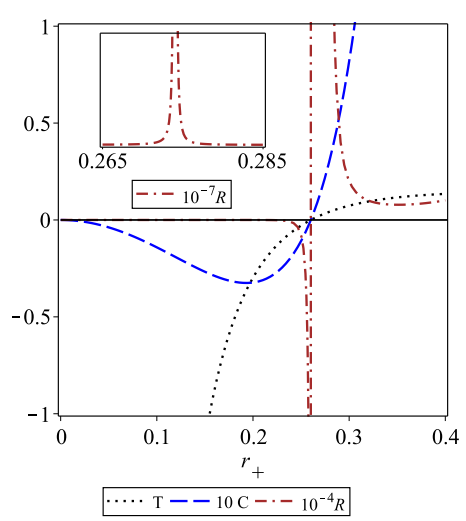

(c)

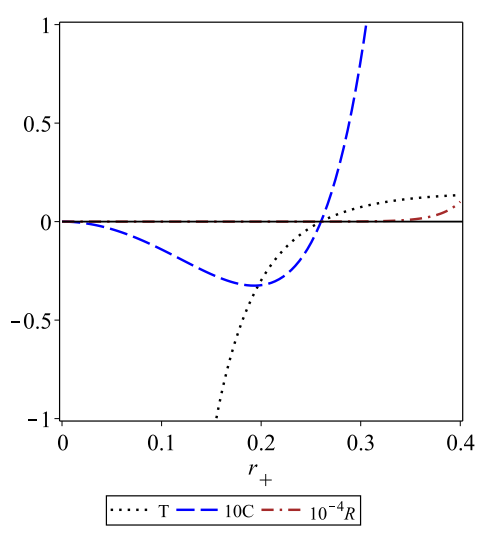

(e)

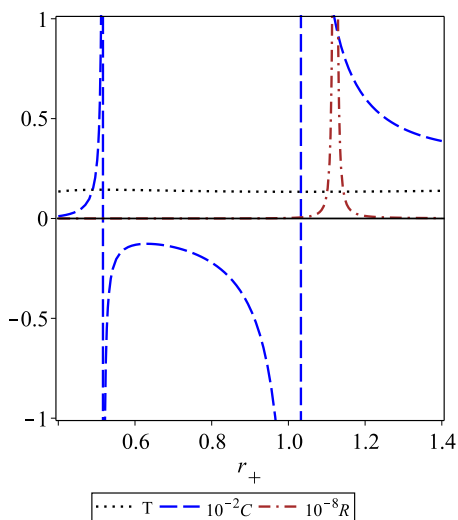

(b)

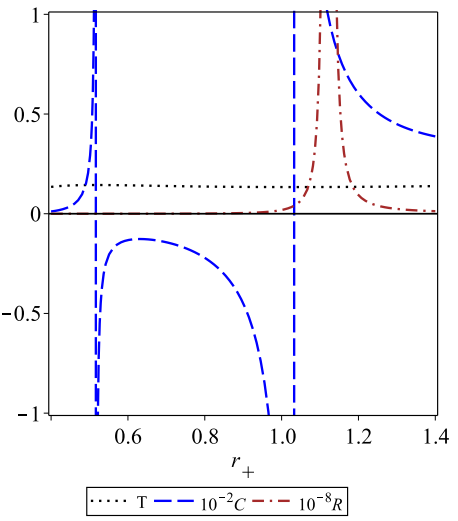

(d)

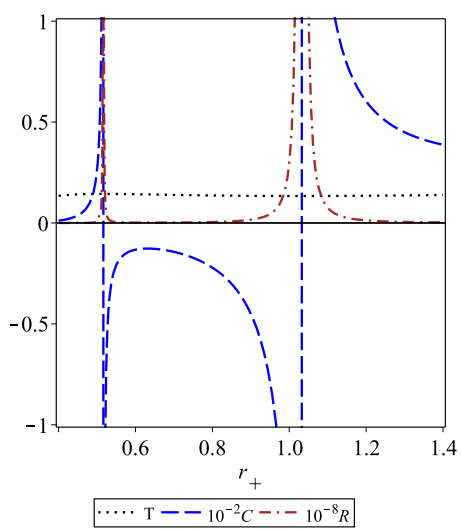

(f)

FIG. 6: $T$ (dotted line), $C_{Q, \mu}$ (dashed line) and Ricci scalar ( dash-dotted line) versus $r_{+}$for $Q=0.2, \mu=0.15$, $\mathcal{B}=0.2, \beta=0.04, \ell=2$ and $A=0.02$. Weinhold's Ricci scalar (up panels), Ruppeiner's Ricci scalar (middle panels) and Quevedo's Ricci scalar (down panels).

A significant point about geometrical methods is that they are based on the grand canonical ensemble foundation. Here, we connect these methods to the heat capacity only for the sake of comparison and check the validity of the results. 

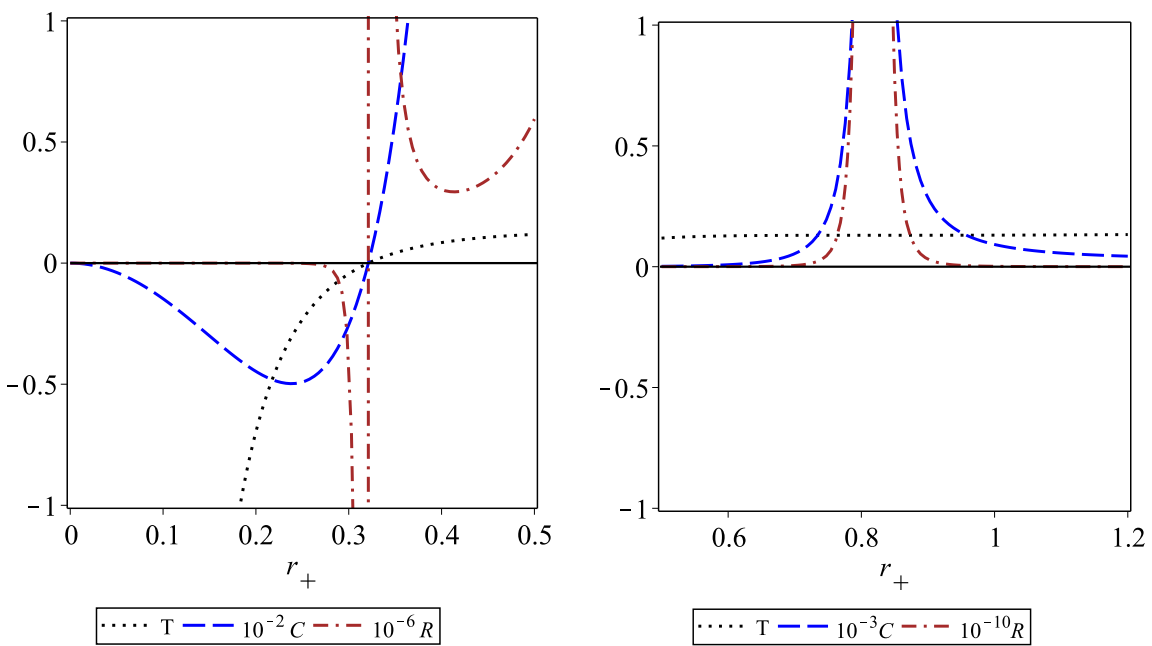

$\mathrm{Q}=0.2502$

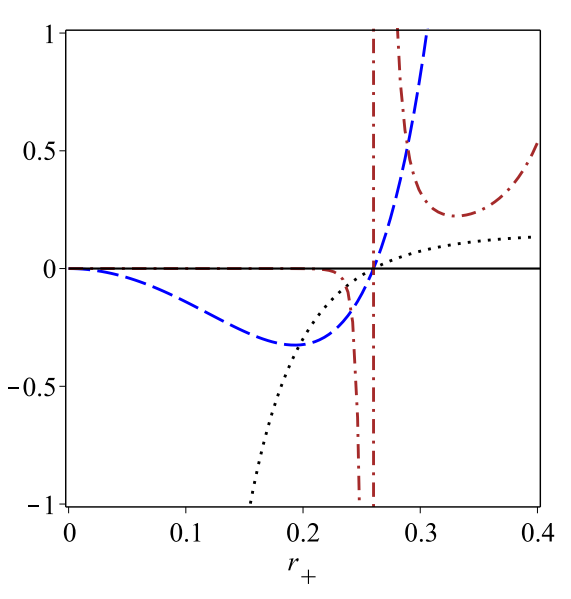

$\cdots \cdots \mathrm{T}--10^{-2} \mathrm{C}-\cdot-10^{-6} R$

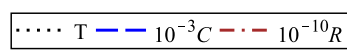

$\mathrm{Q}=0.2502$

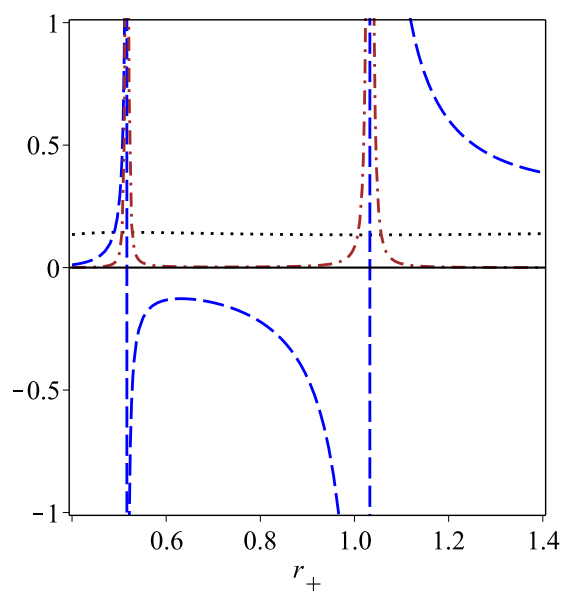

$\mathrm{Q}=0.2$

$\cdots \cdots \mathrm{T}--10^{-2} \mathrm{C}^{-\cdot-} 10^{-9} R$

$\mathrm{Q}=0.2$

FIG. 7: $T$ (dotted line), $C_{Q, \mu}$ (dashed line) and $\mathcal{R}_{H P E M}$ (dash-dotted line) versus $r_{+}$for $Q=0.2, \mu=0.15, \mathcal{B}=0.2$, $\beta=0.04, \ell=2$, and $A=0.02$.

\section{THERMODYNAMICAL STRUCTURE IN EXTENDED PHASE SPACE}

In this section, we extend the phase space by considering the cosmological constant as a thermodynamical quantity and investigate the phase structure of the system. Recently, the idea of variable $\Lambda$ has been attracted a lot of attention [15, 30]. Considering the cosmological constant as a thermodynamical pressure and its conjugate quantity as a thermodynamical volume leads to a new insight into thermodynamical structure and phase transition of the black holes. Here, we study the phase structure of the system through three approaches including heat capacity, thermodynamical geometry and van der Waals like behavior and show that these approaches yield consistent results.

\section{A. $\quad P-V$ criticality of the charged accelerating black holes}

Regarding the normalization-free of the time coordinate, the van der Waals like behavior of such black holes was investigated in Ref. [128]. They considered $A r_{+}$as a constant parameter and studied phase transition of the black hole in the extended phase space. They showed that in order to have a phase transition, one has to consider $A r_{+}=c t e$. In other words, only under a certain condition, there is a small/large black hole phase transition for accelerating black 
holes. Here, we relax this condition and investigate van der Waals like behavior by re-scaling the time coordinate. We determine critical values by using a new method which was introduced in Ref. [127]. To start, we review some basic thermodynamical properties of charged accelerating AdS black holes. The pressure associated with the cosmological constant is given by

$$
P=-\frac{\Lambda}{8 \pi}=\frac{3}{8 \pi \ell^{2}}
$$

Using Eqs. (4), (9) and (21), one can obtain thermodynamical volume which is the conjugate quantity of pressure as

$$
V=\left(\frac{d M}{d P}\right)_{Q, \mu}=\frac{4 \pi}{3 K \alpha}\left(r_{+}^{3}+2 A^{2} r_{+}^{5}+\frac{3 A^{2} r_{+}^{3}}{16 \pi P}+\frac{9 A^{2} r_{+}}{128 \pi^{2} P^{2}}\left(1+\frac{Q^{2} \mathcal{B}^{2}}{\mu^{2} r_{+}^{2}}\right)\right) .
$$

The Helmholtz free energy which is employed to extract information regarding the phase transitions and chemical equilibrium is expressed as [56, 88, 89]

$$
F=M-T S
$$

the thermodynamic equilibrium corresponds to the global minimum of $F$.

To study the van der Waals like phase transition, obtaining the equation of state is necessary. Inserting Eq. (21) into Eq. (10), one can calculate the equation of state as

$$
P=\frac{3\left(1-A^{2} r_{+}^{2}\right)\left[2 \pi T r_{+}\left(2-\beta^{2}+A^{2} Q^{2}\right)+\left(1-\frac{\mathcal{B}^{2} Q^{2}}{\mu^{2} r_{+}^{2}}\right)\left(A^{2} r_{+}^{2}-1\right)\right]}{8 \pi r_{+}^{2}\left(3-A^{2} r_{+}^{2}\right)} .
$$

To have a better understanding of pressure's properties, we find its limiting behaviors as

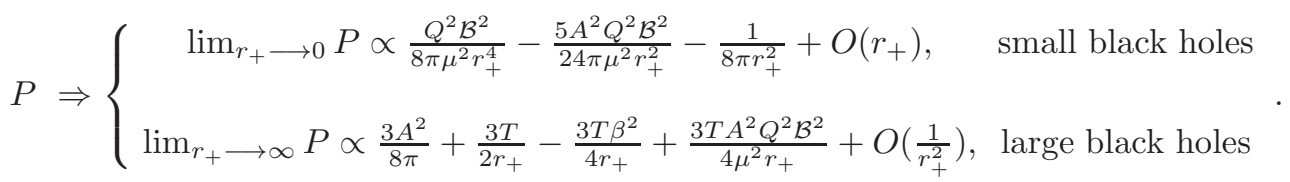

Considering Eq. (25), we find that in the absence of electric charge, the dominant term of the pressure is only a function of the horizon radius that is a negative term. Since the negative pressure is not physically acceptable and it contradicts with the positive definite definition of the pressure in Eq. (21), we conclude that although very small uncharged accelerating black holes have a physical temperature, they are impermissible from the thermodynamic point of view in the extended phase space. While for large black holes, one finds that the acceleration parameter is the governing factor in pressure and depending on the values of different parameters, it can be positive or negative.

Now, we are going to investigate the existence of van der Waals like phase transition for such black holes. To do so, we obtain the specific volume which is related to the horizon radius as

$$
v=2\left(\frac{3 V}{4 \pi}\right)^{\frac{1}{3}}=\frac{2 r_{+}}{K^{\frac{1}{3}}}\left[1+\frac{\beta^{2}}{6}+\frac{A^{2}}{16 \pi P}+\frac{A^{2} r_{+}^{2}}{6}\left(4-\frac{Q^{2} \mathcal{B}^{2}}{\mu^{2} r_{+}^{2}}\right)+\frac{3 A^{2}}{128 \pi^{2} P^{2} r_{+}^{2}}\left(1+\frac{Q^{2} \mathcal{B}^{2}}{\mu^{2} r_{+}^{2}}\right)\right]
$$

and the equation of state (24) can be arranged as

$$
\begin{aligned}
P & =\frac{T \mu^{\frac{1}{3}}}{\mathcal{B}^{\frac{1}{3}} v}-\frac{T \mu^{\frac{1}{3}} \beta^{2}}{3 \mathcal{B}^{\frac{1}{3}} v}+\frac{T A^{2} Q^{2} \mathcal{B}^{\frac{5}{3}}}{3 \mu^{\frac{5}{3}} v}-\frac{\mu^{\frac{2}{3}}}{2 \pi \mathcal{B}^{\frac{2}{3}} v^{2}}-\frac{\beta^{2} \mu^{\frac{2}{3}}}{6 \pi \mathcal{B}^{\frac{2}{3}} v^{2}}+\frac{2 A^{2} \mathcal{B}^{\frac{4}{3}} Q^{2}}{3 \pi \mu^{\frac{4}{3}} v^{2}}+\frac{2 \mathcal{B}^{\frac{2}{3}} Q^{2}}{\pi \mu^{\frac{2}{3}} v^{4}}+\frac{4 Q^{2} \beta^{2} \mathcal{B}^{\frac{2}{3}}}{3 \pi \mu^{\frac{2}{3}} v^{4}} \\
& +\frac{A^{2}}{24 \pi}-\frac{4 A^{2} Q^{4} \mathcal{B}^{\frac{8}{3}}}{3 \pi \mu^{\frac{8}{3}} v^{4}}+\frac{A^{2} Q^{2} \mathcal{B}^{2}}{\pi \chi}+\frac{\mathcal{B} \mu T A^{2} v^{3}}{8 \chi}-\frac{\mathcal{B}^{\frac{2}{3}} \mu^{\frac{4}{3}} A^{2} v^{2}}{8 \pi \chi}-\frac{3 \mathcal{B}^{\frac{4}{3}} \mu^{\frac{8}{3}} A^{2} v^{4}}{8 \pi \chi^{2}}+\frac{3 T \mathcal{B}^{\frac{5}{3}} \mu^{\frac{7}{3}} A^{2} v^{5}}{8 \chi^{2}} \\
& +\frac{3 \mathcal{B}^{\frac{8}{3}} \mu^{\frac{4}{3}} A^{2} Q^{2} v^{2}}{2 \pi \chi^{2}}+\frac{3 T \mu A^{2} Q^{2} \mathcal{B}^{3} v^{3}}{2 \chi^{2}}+\frac{12 A^{2} Q^{4} \mathcal{B}^{4}}{2 \pi \chi^{2}}
\end{aligned}
$$

where

$$
\chi=4 Q^{2} \mathcal{B}^{2}+2 \pi T \mu v^{3} \mathcal{B}-\mu^{\frac{4}{3}} v^{2} \mathcal{B}^{\frac{2}{3}} .
$$


TABLE I: Critical values for $\mathcal{B}=0.2, \beta=0.04, A=0.02$ and $\mu=0.15$.

\begin{tabular}{||c|c|c|c|c||}
\hline \hline$Q$ & $v_{c}$ & $T_{c}$ & $P_{c}$ & $\frac{P_{c} v_{c}}{T_{c}}$ \\
\hline \hline 0.16 & 0.9511 & 0.2041 & 0.0728 & 0.339601 \\
\hline 0.18 & 1.0700 & 0.1814 & 0.0575 & 0.339608 \\
\hline 0.20 & 1.1889 & 0.1633 & 0.0466 & 0.339616 \\
\hline 0.22 & 1.3078 & 0.1484 & 0.0385 & 0.339625 \\
\hline \hline
\end{tabular}

TABLE II: Critical values for $\mathcal{B}=0.2, \beta=0.04, A=0.02$ and $Q=0.2$.

\begin{tabular}{||c|c|c|c|c||}
\hline \hline$\mu$ & $v_{c}$ & $T_{c}$ & $P_{c}$ & $\frac{P_{c} v_{c}}{T_{c}}$ \\
\hline \hline 0.14 & 1.2448 & 0.1524 & 0.0406 & 0.3319 \\
\hline 0.15 & 1.1889 & 0.1633 & 0.0466 & 0.3396 \\
\hline 0.16 & 1.1388 & 0.1742 & 0.0530 & 0.3469 \\
\hline 0.17 & 1.0937 & 0.1851 & 0.0599 & 0.3540 \\
\hline \hline
\end{tabular}

As we know, the van der Waals liquid-gas system goes under a first order phase transition for temperatures smaller than the critical temperature $\left(T<T_{c}\right)$ whereas, its phase transition is a second order one at the critical temperature [15, 129]. Fig. 8, confirms van der Waals like behavior for the charged accelerating AdS black holes. Formation of the swallow-tail shape in $F-T$ diagram (continuous line of Fig. 8) indicates the existence of a first-order small-large black hole transition for $P<P_{c}$. The critical point of the system which coincides with the inflection point of $P-v$ diagram is obtained as

$$
\left.\frac{\partial P}{\partial v}\right|_{v=v_{c}, T=T_{c}}=\left.0 \quad \& \quad \frac{\partial^{2} P}{\partial v^{2}}\right|_{v=v_{c}, T=T_{c}}=0
$$

Eq. (27) is much complicated to determine critical quantities analytically by usual method. But, one can obtain approximate critical values for very small $Q$ and $A$ as follows

$$
\begin{aligned}
& v_{c}=\frac{2 \sqrt{6} Q \mathcal{B}^{\frac{2}{3}} \sqrt{3\left(1+\frac{2 \beta^{2}}{3}\right)-\frac{2 A^{2} Q^{2} \mathcal{B}^{2}}{\mu^{2}}}}{\mu^{\frac{2}{3}} \sqrt{3\left(1+\frac{\beta^{2}}{3}\right)-\frac{4 A^{2} Q^{2} \mathcal{B}^{2}}{\mu^{2}}}}, \\
& T_{c}=\frac{8\left[\frac{2 A^{2} Q^{2} \mathcal{B}^{2}}{\mu^{2}}-3\left(1+\frac{2 \beta^{2}}{3}\right)\right] Q^{2} \mathcal{B}^{2}+\mu^{\frac{4}{3}} v_{c}^{2}\left(3\left(1+\frac{\beta^{2}}{3}\right)-\frac{4 A^{2} Q^{2} \mathcal{B}^{2}}{\mu^{2}}\right) \mathcal{B}^{\frac{2}{3}}}{\pi \mu v_{c}^{3}\left(3\left(1-\frac{\beta^{2}}{3}\right)+\frac{A^{2} Q^{2} \mathcal{B}^{2}}{\mu^{2}}\right) \mathcal{B}}, \\
& P_{c}=\frac{12 \mu^{2} v_{c}^{2}\left(1+\frac{\beta^{2}}{3}\right)+A^{2} v_{c}^{4} \mathcal{B}^{\frac{2}{3}} \mu^{\frac{4}{3}}-16 Q^{2} \mathcal{B}^{\frac{4}{3}}\left(3 \mu^{\frac{2}{3}}\left(3+2 \beta^{2}\right)+v_{c}^{2} A^{2} \mathcal{B}^{\frac{2}{3}}-\frac{6 A^{2} Q^{2} \mathcal{B}^{2}}{\mu^{\frac{4}{3}}}\right)}{24 \pi v_{c}^{4} \mathcal{B}^{\frac{2}{3}} \mu^{\frac{4}{3}}} .
\end{aligned}
$$

In tables \and $\amalg$, we show that how critical quantities and universal critical ratio $\left(\frac{P_{c} v_{c}}{T_{c}}\right)$ change under variation of black hole parameters. In order to show the effects of electric charge and string tension on the critical values of phase transition, we have plotted Fig. 9, and presented tables प and II Fig. 9, and table 【. indicate that as $Q$ increases, the critical pressure and temperature decrease, whereas the critical volume and the universal critical ratio increase. As for the effects of string tension, one can see that the critical volume is a decreasing function of this parameter, whereas the critical pressure, temperature and universal critical ratio are an increasing function of this parameter (see Fig. 9, and table II). 


\section{B. Critical points of the charged accelerating black holes via new prescription}

As it was mentioned, one cannot obtain the critical values analytically via the usual method due to the complexity of Eq. (27). Since this method is not practical for black holes with the non-spherical horizon in most gravitational theories [126], we employ an alternative approach for obtaining the critical values. This method is based on the denominator of heat capacity [127]. Using the analogy between pressure and the cosmological constant and solving denominator with respect to pressure, one can obtain a new relation for pressure. It is worthwhile to mention that this new pressure is not the same pressure that was obtained in Eq. (27). Substituting Eqs. (21) and (26) in Eq. (17), the new pressure is obtained as follows

$$
P_{\text {new }}=-\frac{\chi_{2}}{3}+\left(-\frac{w}{2}+\frac{\sqrt{12 \varrho^{3}+81 w^{2}}}{18}\right)^{\frac{1}{3}}+\left(-\frac{w}{2}-\frac{\sqrt{12 \varrho^{3}+81 w^{2}}}{18}\right)^{\frac{1}{3}}
$$

where

$$
\begin{aligned}
\varrho & =-\frac{\chi_{2}^{2}}{3}-\frac{\mu^{\frac{2}{3}} A^{2}\left(1+\frac{v^{2} A^{2} \mathcal{B}^{\frac{2}{3}}}{2 \mu^{\frac{2}{3}}}\right)^{-1}}{16 \pi^{2} v^{2} \mathcal{B}^{\frac{2}{3}}}, \\
w & =\frac{2 \chi_{2}^{3}}{27}+\frac{\mu^{\frac{2}{3}} \chi_{2} A^{2}\left(1+\frac{v^{2} A^{2} \mathcal{B}^{\frac{2}{3}}}{2 \mu^{\frac{2}{3}}}\right)^{-1}}{48 \pi^{2} v^{2} \mathcal{B}^{\frac{2}{3}}}-\frac{3 \mu^{\frac{4}{3}} A^{2}\left(1+\frac{4 Q^{2} \mathcal{B}^{\frac{4}{3}}}{v^{2} \mu^{\frac{4}{3}}}\right)}{32 \pi^{3} v^{4} \mathcal{B}^{\frac{4}{3}}\left(1+\frac{v^{2} A^{2} \mathcal{B}^{\frac{2}{3}}}{2 \mu^{\frac{2}{3}}}\right)}, \\
\chi_{2} & =\frac{-7 A^{2}\left(1-\frac{144 Q^{2} \mathcal{B}^{\frac{2}{3}}}{7 \mu^{\frac{2}{3}} v^{4} A^{2}}\right) \mathcal{B}^{\frac{2}{3}}-\frac{4 \mu^{\frac{2}{3}}}{v^{2}}\left(\left(3+\beta^{2}\right)-\frac{2 A^{2} Q^{2} \mathcal{B}^{2}}{\mu^{2}}\right)}{24 \pi\left(1+\frac{v^{2} A^{2} \mathcal{B}^{\frac{2}{3}}}{2 \mu^{\frac{2}{3}}}\right) \mathcal{B}^{\frac{2}{3}}} .
\end{aligned}
$$

Replacing Eqs. (26) and (30) in Eq. (10), one can obtain a new relation for the temperature which is independent of pressure. The new pressure and temperature have a maximum point that exactly coincides with the inflection point of $P-v$ and $T-v$ diagram. In other words, these maximum pressure and temperature are the same critical pressure and temperature and their proportional volume is critical volume (see dashed lines in left and middle panels of Fig. (8).

It should be noted that the black dashed curve in Fig. 8 is not representing the boundary between small black hole and large black hole. This curve is the same spinodal curve. In fact the first-order phase boundary is detected by the binodal curve or coexistence curve which has been illustrated in Fig. 10. The region between spinodal and binodal curves is related to the metastable black holes which is equivalence to the positive heat capacity. The region under the binodal curve indicates small black hole+large black hole which are thermodynamically unstable.

As it was mentioned, for pressures and temperatures smaller than the critical pressure and temperature, one can observe a first order small/large black hole phase transition. By looking at $P-v$ and $T-v$ diagrams in Fig. 11 (up panels), one can find that for large electric charges, pressure (temperature) is only a decreasing (an increasing) function of the volume without any extremum. For $F-T$ diagram, evidently, free energy is a decreasing function of the temperature and swallow-tail shape does not appear. In this case, just a single stable phase exists for black holes. For a specific value of $Q$, one can observe an extremum that separates two small and large stable phases from each other. As for small values of $Q$, two extrema are formed in $P-v$ and $T-v$ diagrams. In this case, three phases exist which are small, medium and large black holes. The region between two extrema is related to medium black holes which are unstable. Whereas, the regions before the first extremum and after the second extremum in $P-v$ and $T-v$ diagrams are related to small and large phases respectively. Also, $P-v$ diagram shows that as $Q$ decreases, the pressure related to phase transition points decreases. Since, pressure is related to the cosmological constant which is related to asymptotical curvature of the background, one can say that as $Q$ decreases the necessity of having a background with higher curvature decreases. Up middle and right panels of Fig. 11 show that as $Q$ decreases, the temperature related to phase transition points increases and the difference between free energy of different phases grows larger. So, rapidly accelerating black holes need to absorb more mass in order to have phase transition. In other words, by decreasing the electric charge, the system achieves a stable state barely.

Taking a closer look at down panels of Fig. 11, one can find that the effect of string tension is the opposite of that of the electric charge. Also, investigating the variation of string tension on the phase structure of the system shows 

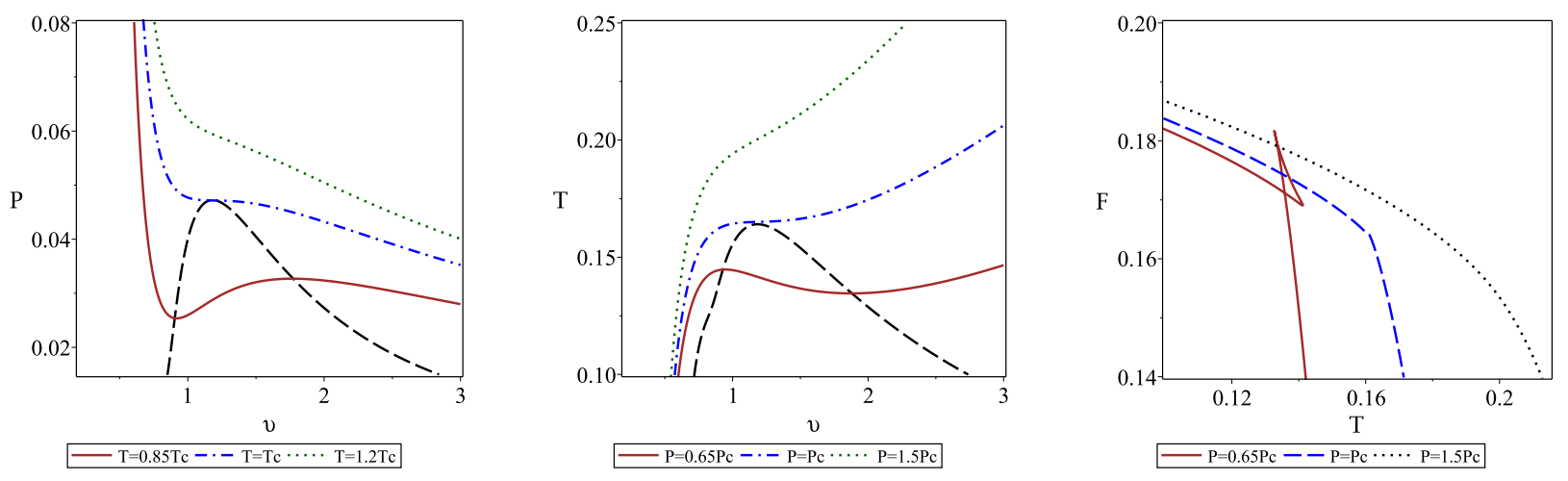

FIG. 8: van der Waals like phase diagrams for $\mathcal{B}=0.2, \beta=0.04, A=0.02, \mu=0.15$ and $Q=0.2$. Left panel: $P-v$ diagram for $T=0.14$ (continuous line), $T=0.164$ (dash-dotted line), $T=0.2$ (dotted line) and $P_{n e w}$ (dashed line). Middle panel: $T-v$ diagram for $P=0.03$ (continuous line), $P=0.048$ (dash-dotted line), $P=0.07$ (dotted line) and $T_{\text {new }}$ (dashed line). Right panel: $F-T$ diagram for $P=0.03$ (continuous line), $P=0.048$ (dash-dotted line) and $P=0.07$ (dotted line).
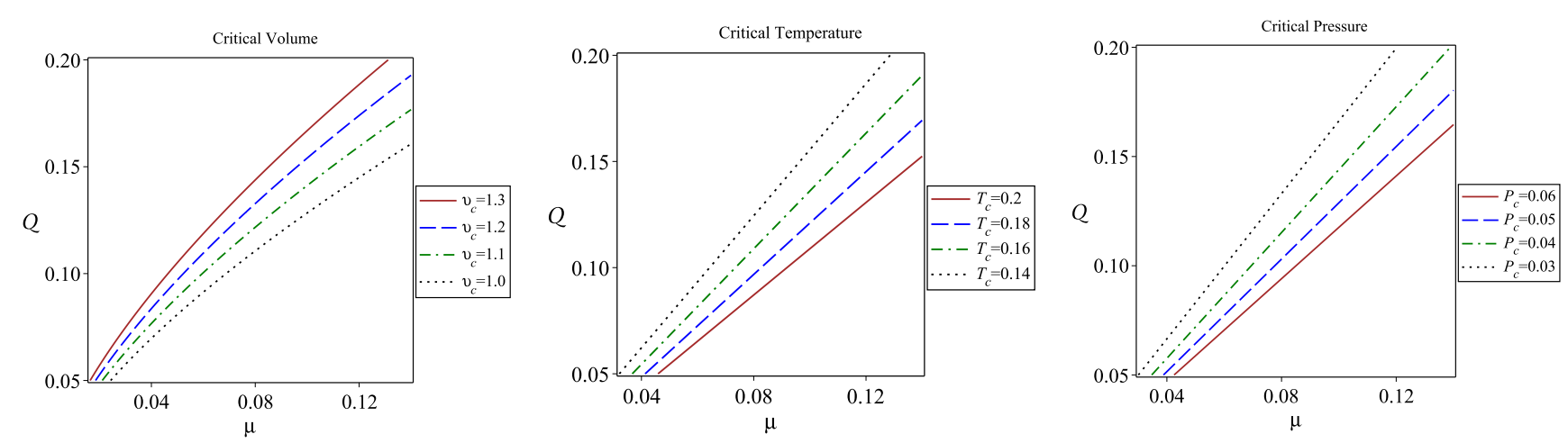

FIG. 9: Variation of the critical values as functions of black hole parameters for $\mathcal{B}=0.2, \beta=0.04$ and $A=0.02$.

that as $\mu$ increases, the distance between two extrema increases. This shows that a stable small or large charged accelerating black hole goes to an unstable phase by increasing this parameter. Since string tension is linearly related to the conical deficit, the larger the tension, the steeper the cone (see Eq. 8). So, it is expected that a small/large charged accelerating black hole exits in its stable state if it is pulled by more powerful string tension.
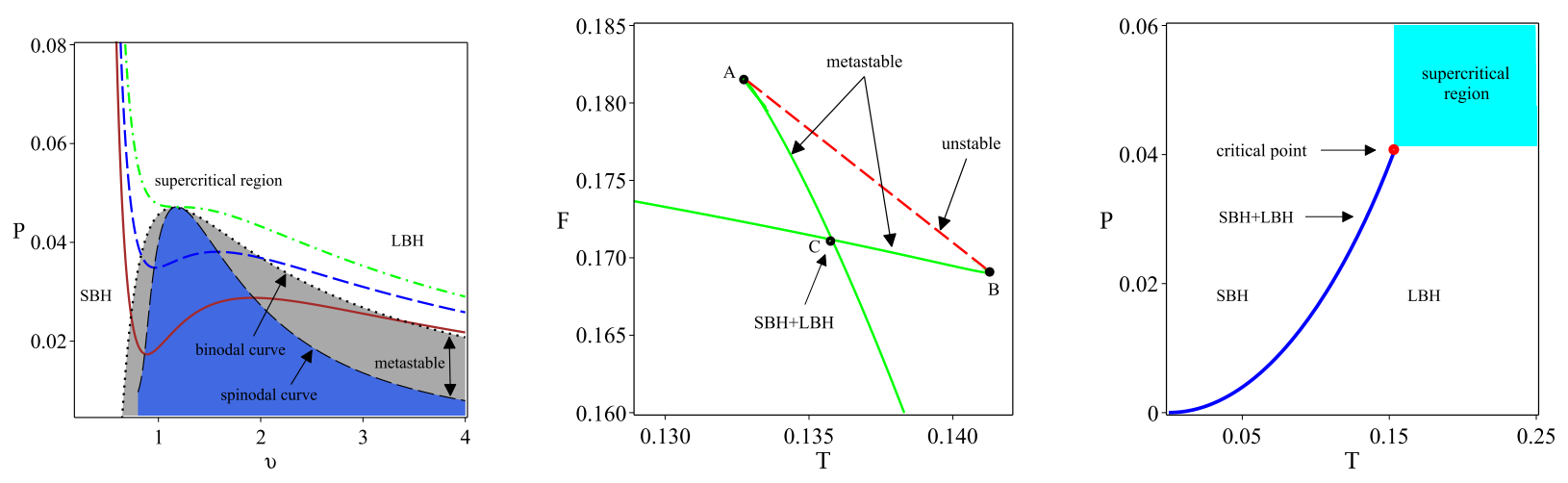

FIG. 10: $P-v, F-T$ and $P-T$ diagram for $\mathcal{B}=0.2, \beta=0.04, A=0.02, \mu=0.15$ and $Q=0.2$. 


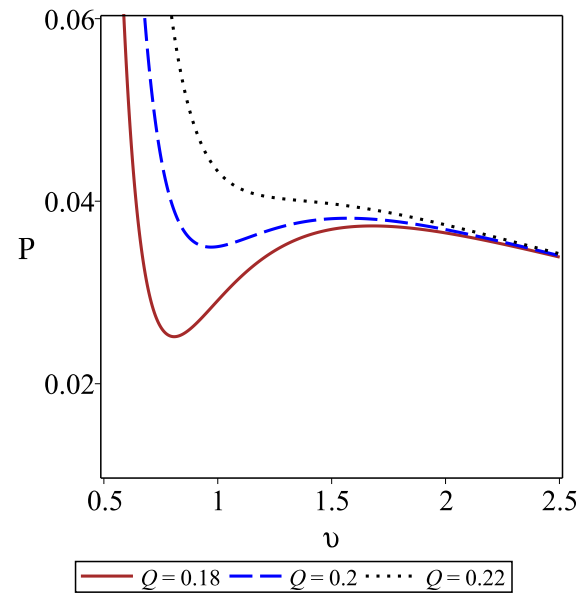

(a)

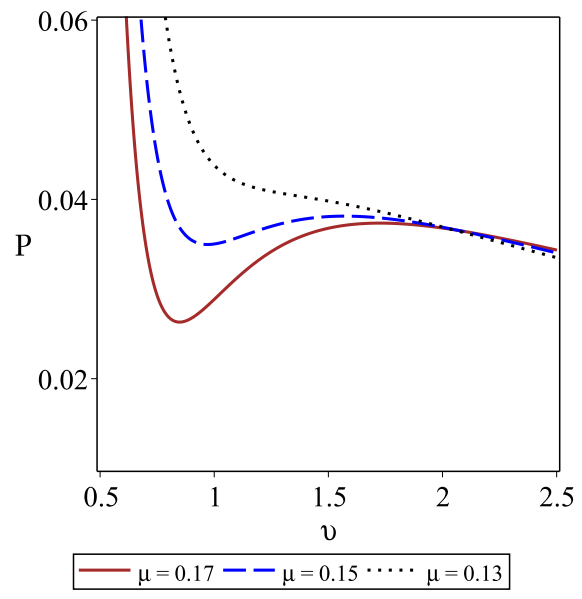

(d)

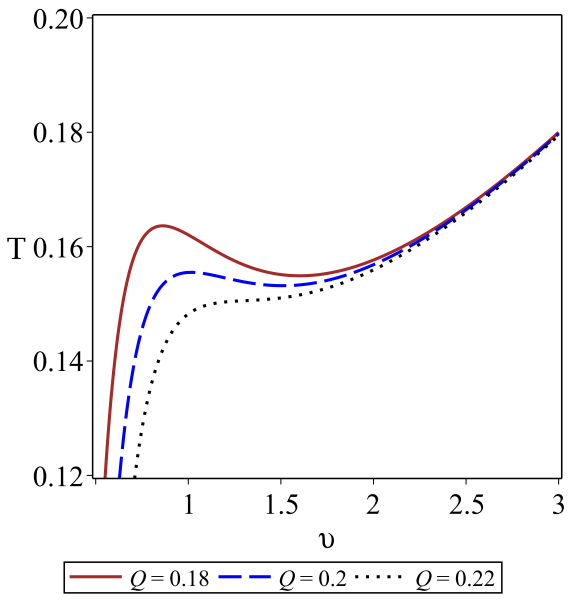

(b)

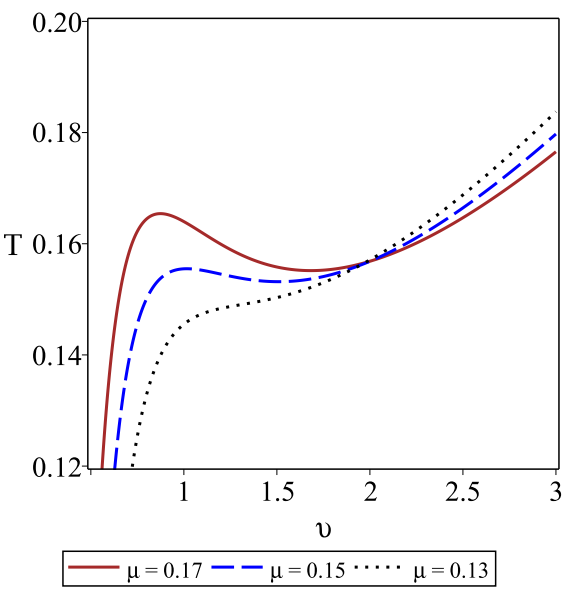

(e)

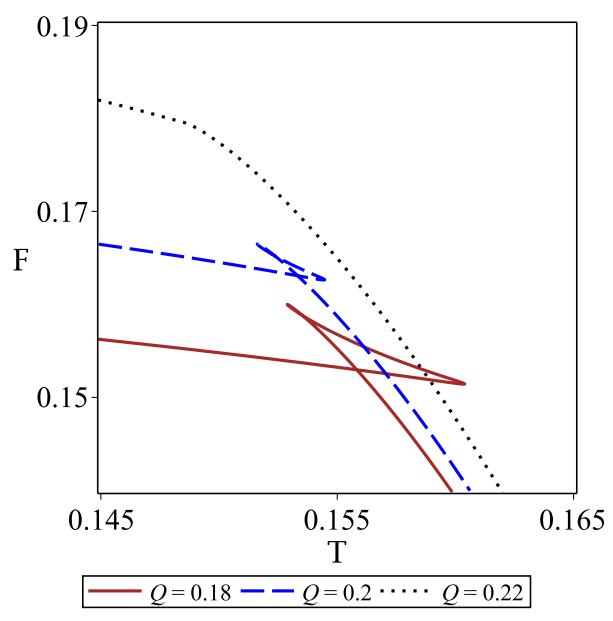

(c)

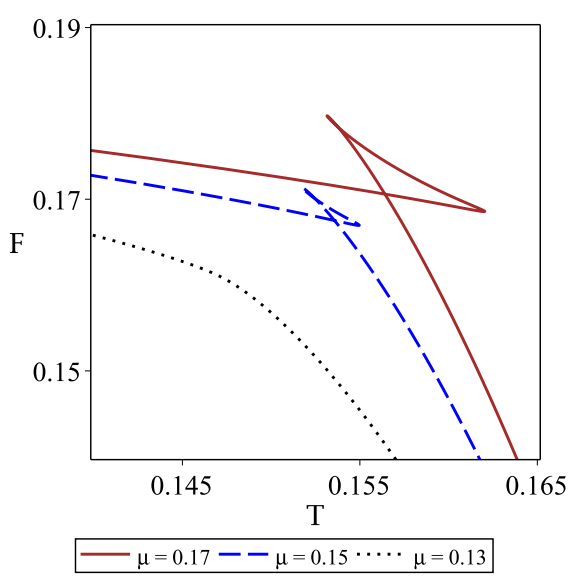

(f)

FIG. 11: For $\mathcal{B}=0.2, \beta=0.04$ and $A=0.02 . T=0.9 T_{c}$ for $P-v$ diagram and $P=0.85 P_{c}$ for $T-v$ and $F-T$ diagrams. Up panels: for $\mu=0.15$ and different values of the electric charge. Down panels: for $Q=0.2$ and different values of the string tension.

\section{Geometrical thermodynamics in the extended phase space}

Here, we employ GTs to study phase transition in the extended phase space. As it is known, the cosmological constant is related to thermodynamical pressure in the extended phase space. Here, we consider the pressure as an extensive parameter and explore its effects on different approaches of thermodynamical geometry. In this case, thermodynamical space transforms from $M(S, Q)$ to $M(S, Q, P)$. So, the Weinhold, Ruppeiner, Quevedo and HPEM 
metrics are modified as

$$
d s^{2}=\left\{\begin{array}{cc}
M g_{a b}^{W} d X^{a} d X^{b}, & \text { Weinhold } \\
-\frac{M}{T} g_{a b}^{R} d X^{a} d X^{b}, & \text { Ruppeiner } \\
\left(S M_{S}+Q M_{Q}+P M_{P}\right)\left(-M_{S S} d S^{2}+M_{Q Q} d Q^{2}+M_{P P} d P^{2}\right), & \text { Quevedo I . } \\
S M_{S}\left(-M_{S S} d S^{2}+M_{Q Q} d Q^{2}+M_{P P} d P^{2}\right), & \text { Quevedo II } \\
\frac{S M_{S}}{M_{Q Q}^{3} M_{P P}^{3}}\left(-M_{S S} d S^{2}+M_{Q Q} d Q^{2}+M_{P P} d P^{2}\right), & \text { HPEM }
\end{array}\right.
$$

By calculating the Ricci scalar of these metrics, one can obtain the denominator of the Ricci scalar with the following forms

$$
\operatorname{denom}(\mathcal{R})=\left\{\begin{array}{c}
-2 M^{3}\left(M_{Q P}^{2} M_{S S}+M_{S Q}^{2} M_{P P}+M_{S P}^{2} M_{Q Q}-M_{S S} M_{Q Q} M_{P P}-2 M_{S Q} M_{S P} M_{Q P}\right)^{2}, \quad \text { Weinhold } \\
-2 M^{3} T^{3}\left(M_{Q P}^{2} M_{S S}+M_{S Q}^{2} M_{P P}+M_{S P}^{2} M_{Q Q}-M_{S S} M_{Q Q} M_{P P}-2 M_{S Q} M_{S P} M_{Q P}\right)^{2}, \\
2 M_{S S}^{2} M_{Q Q}^{2} M_{P P}^{2}\left(S M_{S}+Q M_{Q}+P M_{P}\right)^{3}, \quad \text { Queveiner I . } \\
2 S^{3} M_{S S}^{2} M_{Q Q}^{2} M_{P P}^{2} M_{S}^{3}, \\
S^{3} M_{S}^{3} M_{S S}^{2},
\end{array}\right.
$$

Now, we investigate thermodynamical behavior of the system through geometrical approaches. As it is observed in Fig. 12, both Weinhold and Ruppeiner metrics fail to produce consistent results with phase transition points (see up panels of Fig. 12). In the case of Quevedo's metrics, Quevedo I does not include bound point in divergencies of its Ricci scalar and it can only describe phase transition points. As for Quevedo II, although some of its divergencies coincide with bound and phase transition points, similar to the case of Quevedo I, it has an extra divergency that does not match with any phase transition point (see middle and down panels of Fig. 12). Regarding the HPEM's metric, its Ricci scalar has two divergencies at $P=P_{c}$. One divergency coincides with the root of heat capacity and the other one matches with divergency of the heat capacity (see Fig. 13r). For the case of $P<P_{c}$, three divergencies are observed: one of these divergencies is related to the root of heat capacity and the other ones are coincident with divergencies of the heat capacity (see up panels of Fig. 13). As for $P>P_{c}$, HPEM's Ricci scalar has only a divergence point which is located at the root of heat capacity (Fig. 13d). These results show that the HPEM's metric can be considered a powerful tool to describe the phase structures of such black holes.

As we have already mentioned, one can employ the Legendre invariant metrics for probing molecular interaction during the phase transition as well 69]. Since the HPEM's metric could provide a precise picture of phase transitions, we employ this method and check whether it can describe microscopic properties of the system. As we see from Fig. 13., scalar curvature goes to negative infinity at $P=P_{c}$, implies an attractive interaction between the microscopic molecules. Regarding Fig. 13b for $P<P_{c}$, it is clear that the scalar curvature is negative around both divergence points. Taking a close look at this figure, one can find that scalar curvature becomes positive near the smaller divergence point which shows that a weak repulsive interaction dominates for small black holes. It is worthwhile to mention that these results are similar to those obtained for van der Waals fluid [130]. So, HPEM's metric can describe phase structure and microstructure of the black hole, simultaneously. Here, we should point out that we consider both the numerator and denominator of $R_{H}$ to draw Fig. 13, while in Fig. 12, we only considered denominators of Ricci scalars. Since other metrics have not been successful in investigating phase transitions, we have not used them for studying microscopic properties.

In 2017, two new metrics were introduced by geometric interpretation of criticality conditions [77, 78]. In the next section, we employ these two metrics and show that they are not suitable candidates for accelerating cases in which thermodynamical volume is not a linear function of the horizon radius. 


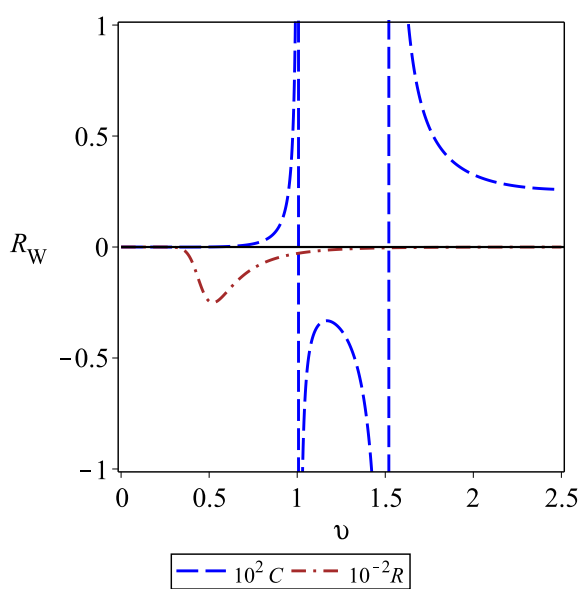

(a)

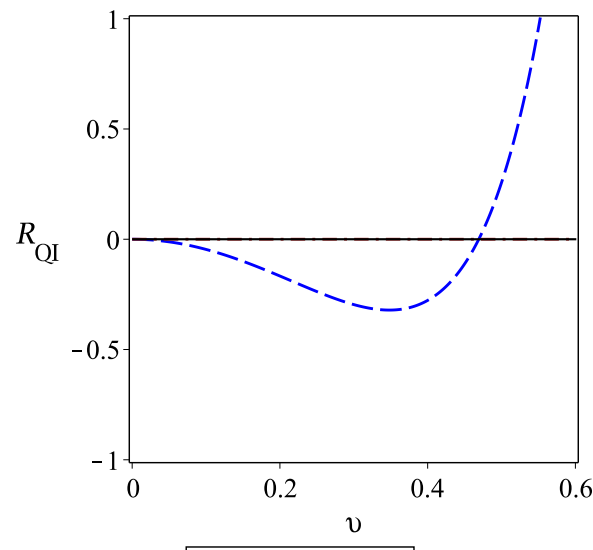

(c)

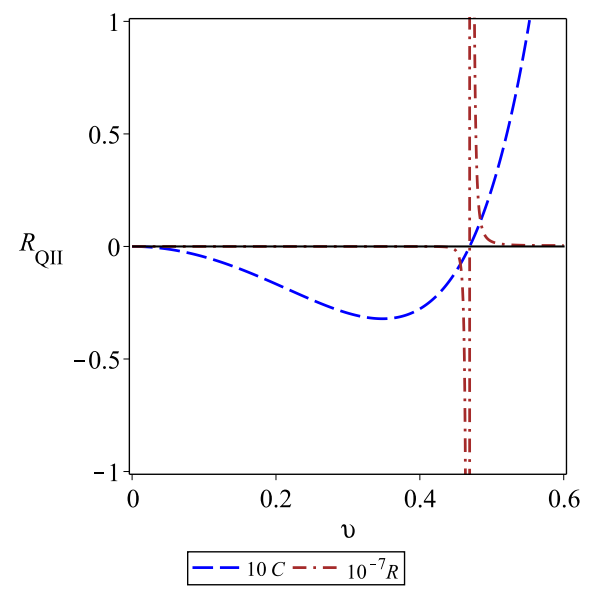

(e)

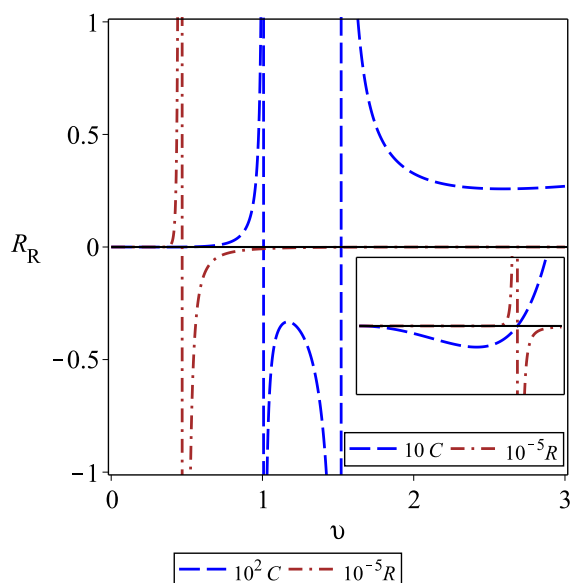

(b)

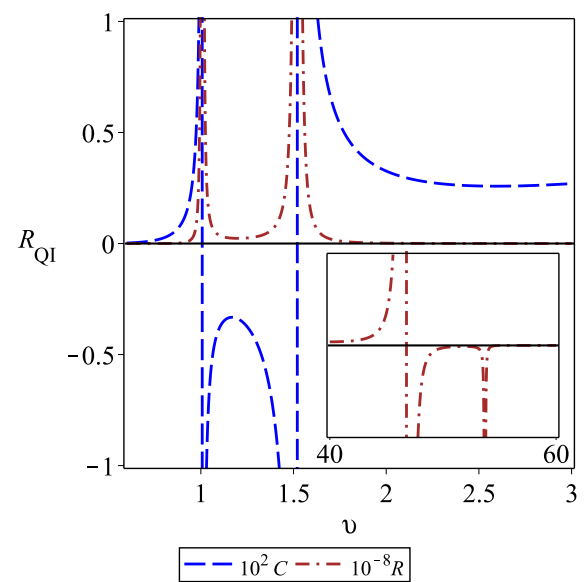

(d)

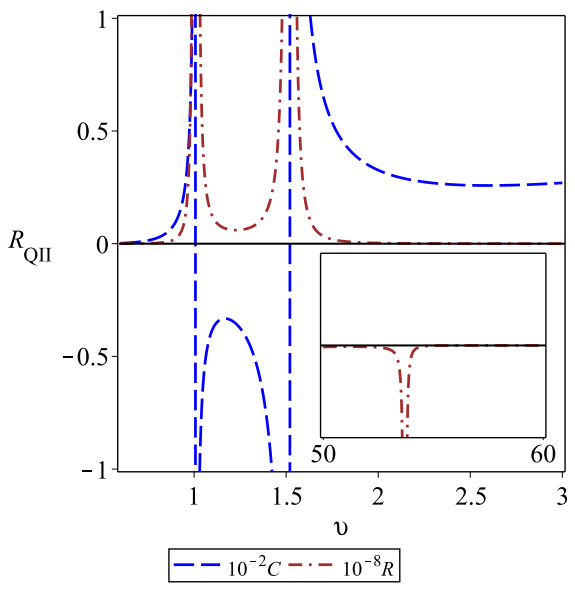

(f)

FIG. 12: $C_{P, Q, \mu}$ (dashed line) and Ricci scalar (dash-dotted line) versus $v$ for $\mathcal{B}=0.2, \beta=0.04, A=0.02, \mu=0.15$, $Q=0.2$ and $P=0.85 P_{c}$. Weinhold's Ricci scalar (up-left panel), Ruppeiner's Ricci scalar (up-right panel), Quevedo's Ricci scalar (middle and down panels). 


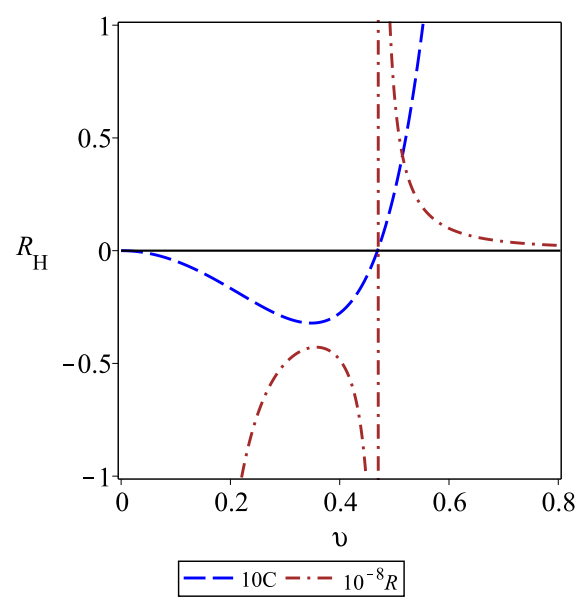

(a) $P=0.85 P_{c}$

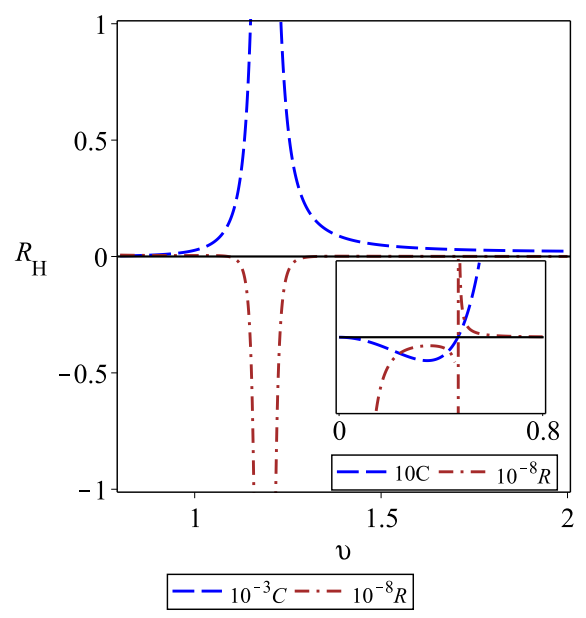

(c) $P=P_{c}$

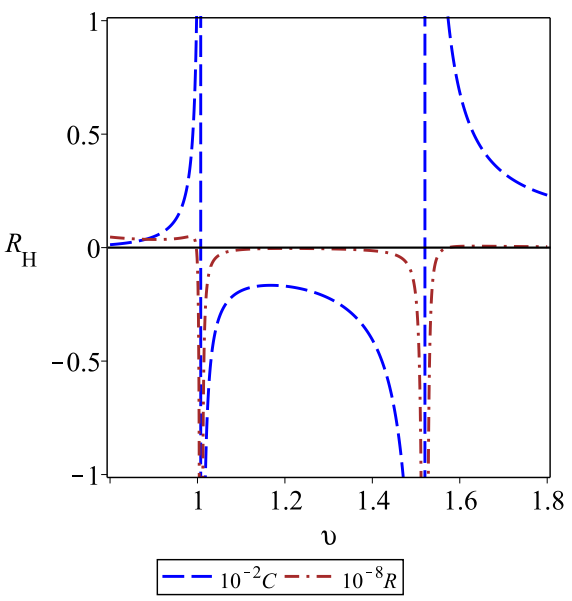

(b) $P=0.85 P_{c}$

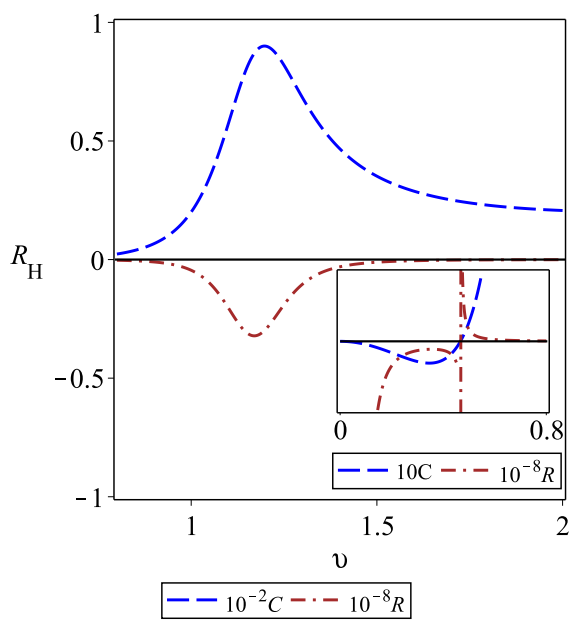

(d) $P=1.1 P_{c}$

FIG. 13: $C_{P, Q, \mu}$ (dashed line) and $\mathcal{R}_{H P E M}$ (dash-dotted line) versus $v$ for $\mathcal{B}=0.2, \beta=0.04, A=0.02, \mu=0.15$ and $Q=0.2$.

\section{1. geometrical description of critical conditions}

In this method, two independent GT metrics are defined by the geometrical description of two critical conditions where the defined metrics are invariant under Legendre transformations. Ricci scalars of these metrics diverge at the critical point. For the first condition, the Helmholtz free energy is considered as the appropriate quantity to define GT metric. Whereas, for the geometrical description of the second condition, the pressure is a proper thermodynamical quantity (instead of F). Now, we employ these two metrics for studying phase transition points of charged accelerating AdS black hole.

In order to obtain first thermodynamic metric, one can define a thermodynamical phase space with the coordinates $Z^{A}=\left\{F, \xi^{a}, \rho^{a}\right\}$ where $\xi^{a}=\{V, T, Q\}$ are the thermodynamical variables and $\rho^{a}=\left\{F_{V}, F_{T}, F_{Q}\right\}$ are the conjugate quantities corresponding to these variables which are defined as follow

$$
F_{V}=\left(\frac{\partial F}{\partial V}\right)_{T, Q}=-P \quad \& \quad F_{T}=\left(\frac{\partial F}{\partial T}\right)_{V, Q}=-S \quad \& \quad F_{Q}=\left(\frac{\partial F}{\partial Q}\right)_{V, T}=\Phi
$$

In this case, the Legendre invariant metric is expressed as

$$
g_{1}^{(P V)}=(-P V-S T+\Phi Q)\left(-F_{V V} d V^{2}+F_{T T} d T^{2}+F_{Q Q} d Q^{2}+2 F_{T Q} d T d Q\right) .
$$


The metric coefficients can be identified as [78]

$$
\begin{aligned}
& f(V, T, Q)=F_{V V}\left(V F_{V}+T F_{T}+Q F_{Q}\right), \\
& h(V, T, Q)=F_{T T}\left(V F_{V}+T F_{T}+Q F_{Q}\right), \\
& x(V, T, Q)=F_{Q Q}\left(V F_{V}+T F_{T}+Q F_{Q}\right), \\
& y(V, T, Q)=F_{T Q}\left(V F_{V}+T F_{T}+Q F_{Q}\right) .
\end{aligned}
$$

Straightforward calculation shows that the denominator of the Ricci scalar can be written as

$$
\operatorname{denom}\left(R_{1}\right)=\frac{1}{2 f^{2}\left(h x-y^{2}\right)^{2}} .
$$

Now, we consider the pressure as a proper thermodynamical quantity. In this case, a thermodynamical phase space is defined with the coordinates $Z^{A}=\left\{P, \xi^{a}, \rho^{a}\right\}$ where $\xi^{a}=\{V, T, Q\}$ and $\rho^{a}=\left\{P_{V}, P_{T}, P_{Q}\right\}$ are the variables and conjugate quantities, respectively. The geometrical metric in the invariant $\theta_{P}$ picture is given by

$$
g_{2}^{(P V)}=\left(V P_{V}+T P_{T}+Q P_{Q}\right)\left(-P_{V V} d V^{2}+P_{T T} d T^{2}+P_{Q Q} d Q^{2}+2 P_{T Q} d T d Q\right)
$$

with following metric coefficients

$$
\begin{aligned}
& \omega(V, T, Q)=P_{V V}\left(V P_{V}+T P_{T}+Q P_{Q}\right), \\
& \beta(V, T, Q)=P_{T T}\left(V P_{V}+T P_{T}+Q P_{Q}\right), \\
& \gamma(V, T, Q)=P_{Q Q}\left(V P_{V}+T P_{T}+Q P_{Q}\right), \\
& \varepsilon(V, T, Q)=P_{T Q}\left(V P_{V}+T P_{T}+Q P_{Q}\right) .
\end{aligned}
$$

By calculating the Ricci scalar, one can obtain the following expression for its denominator

$$
\operatorname{denom}\left(R_{2}\right)=\frac{1}{2 \omega^{2}\left(\beta \gamma-\varepsilon^{2}\right)^{2}} .
$$

We draw behavior of $R_{1}$ and $R_{2}$ with respect to $v$ in Fig. 14. One can see that $R_{1}$ and $R_{2}$ have an extra divergence point at $T=T_{c}$. For $R_{2}$, one of these divergencies is exactly coincident with divergency of the heat capacity at the critical temperature. But the other ones do not match with heat capacity's divergence points (see right panel of Fig. 14). As for $R_{1}$, none of its divergencies coincide with heat capacity's divergency (see left panel of Fig. 14). So, this method cannot provide an appropriate picture of phase transition for charged accelerating AdS black holes.

\section{CONCLUSION}

In this paper, we considered the thermodynamical behavior of the charged accelerating AdS black holes and investigated their thermal stability and phase transition by calculating the heat capacity in canonical ensemble. First, we studied thermodynamical structure of these black holes in the non-extended phase space and showed that the electric charge, AdS radius and string tension are important factors for observing phase transition. We found a relation between the electric charge, string tension and AdS radius to indicate that the condition $\frac{Q \mathcal{B}}{\mu \ell}<0.1667$, should be satisfied in order to have phase transition for such black holes. Studying the effects of electric charge and string tension on stability conditions showed that as string tension (electric charge) increases (decreases), the region of instability increases.

In addition, we employed GT method to investigate phase transition of the system. We found that among wellknown thermodynamical metrics, only HPEM one provided consistent results with the heat capacity and was able to describe both bound and phase transition points simultaneously. Also, we saw that the behavior of HPEM's Ricci scalar around its divergence points was different for the corresponding root and divergence points of the heat capacity. 

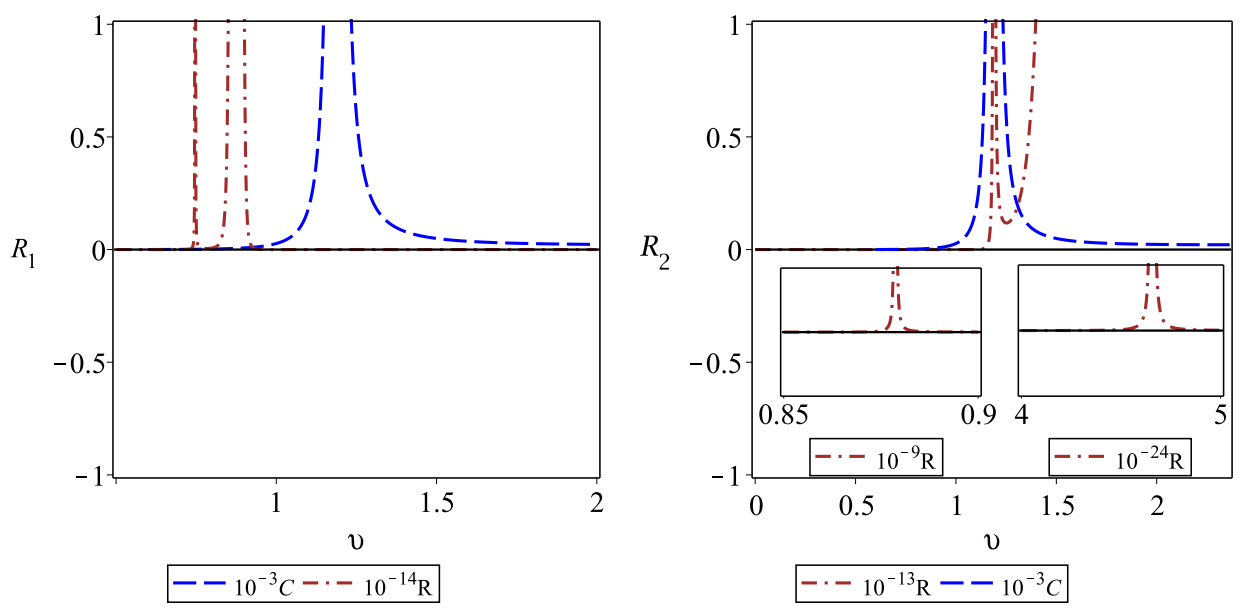

FIG. 14: $C_{P, Q, \mu}$ (dashed line) and $R$ (dash-dotted line) versus $v$ for $\mathcal{B}=0.2, \beta=0.04, Q=0.2, \mu=0.15, A=0.02$ and $T=T_{c}$.

In other words, by (un)changing the sign of HPEM's Ricci scalar around its divergence points, one can recognize bound and phase transition points from each other.

Next, we extended the phase space by considering analogy between the cosmological constant and thermodynamical pressure, and studied van der Waals like phase transition. We also used the denominator of the heat capacity and obtained a new relation for pressure which its maximum was the same critical pressure. Substituting the new pressure in temperature, we obtained a new relation for the temperature contains a maximum which was exactly coincident with the critical temperature. Investigating the effects of electric charge and string tension, we found that the critical pressure and temperature are decreasing (increasing) functions of electric charge (string tension). Whereas, the critical volume is an increasing (a decreasing) function of electric charge (string tension).

Studying $T-v$ and $F-T$ diagrams showed that for small (large) values of $Q(\mu)$, phase transitions take place in higher temperatures. This indicates that obtaining a stable state becomes more difficult for these black holes and they need to absorb more mass from surrounding in order to have a phase transition. Also, studying $P-v$ diagram indicated that as electric charge (string tension) increases (decreases), the pressure related to the phase transition increases which shows that the necessity of having a background with higher curvature increases. Also, we found that as $\mu$ increases, the distance between two extrema increases. This revealed the fact that a small/large accelerating black hole exits in its stable state by increasing this parameter.

Finally, we employed well-known thermodynamical metrics for studying the critical behavior of the system. We noticed that similar to the non-extended case, just divergencies of HPEM's Ricci scalar were exactly coincident with bound and phase transition points. We also used the geometrical approach which was obtained by the geometrical description of critical conditions. We saw that the Ricci scalar included an extra divergence point at the critical temperature and was not able to describe the critical point. So, only the HPEM's metric could provide an appropriate picture of phase transition for accelerating AdS black holes in both extended and non-extended phase space.

\section{Acknowledgments}

SHH thanks Shiraz University Research Council. The work of BEP has been supported financially by the Research Institute for Astronomy and Astrophysics of Maragha (RIAAM) under research project No. 1/6025-58.

\section{Appendix \\ A: normalization of the time coordinate}

The action, including boundary counterterms [131 133], is

$$
I=\frac{1}{16 \pi} \int_{M} d^{4} x \sqrt{g}\left[R+\frac{6}{\ell^{2}}-F_{a b} F^{a b}\right]+\frac{1}{8 \pi} \int_{\partial M} d^{3} x \sqrt{h}\left[\mathcal{K}-\frac{2}{\ell}-\frac{\ell}{2} \mathcal{R}(h)\right],
$$


where $F_{a b}$ is the electromagnetic field tensor and $h_{a b}$ is the intrinsic metric on $\partial M$. $\mathcal{K}$ and $\mathcal{R}(h)$ are, respectively, the extrinsic curvature and Ricci scalar of the boundary. Varying the action gives the energy momentum tensor as

$$
8 \pi \mathcal{T}_{a b}=\ell \mathcal{G}_{a b}(h)-\frac{2}{\ell} h_{a b}-\mathcal{K}_{a b}+h_{a b} \mathcal{K} .
$$

To compute the quantities appearing in this expression, we require new coordinates near the boundary of AdS, typically parameterized by Fefferman-Graham coordinates, in which the geometry takes a standard format [83, 87]

$$
d s^{2}=\frac{\ell^{2}}{z^{2}} d z^{2}+z^{-2}\left(\gamma_{a b}^{0}+z^{2} \gamma_{a b}^{2}+\ldots\right) d x^{a} d x^{b} .
$$

One can perform an asymptotic expansion for the coordinate transformation as

$$
\frac{1}{r}=-A \xi-\sum_{n=1}^{4} X_{n}(\xi) z^{n}, \quad \& \quad \cos \theta=\xi+\sum_{n=1}^{4} Y_{n}(\xi) z^{n}
$$

The functions $X_{n}$ and $Y_{n}$ are fixed by requiring the metric (1) be of the form Eq. (40). By a systematic expansion, one can determine the $Y_{n}$ in terms of the $X_{n}$. For example, at leading order, $\mathcal{O}\left(z^{-2}\right)$

$$
Y_{1}(\xi)=-\frac{A \ell^{2} X_{1}(\xi) G(\xi)}{F^{2}(\xi)}, \quad \& \quad X_{1}(\xi)=-\frac{F(\xi)^{3}}{\alpha \omega(\xi)},
$$

which elucidates the conformal degree of freedom in the boundary metric, $\omega$, with

$$
G(x)=\left(1-x^{2}\right)\left(1+2 m A x+e^{2} A^{2} x^{2}\right), \quad \& \quad F(x)=\sqrt{1-A^{2} \ell^{2} G(x)} .
$$

The expression (42) ensures $g_{z z}=\frac{\ell^{2}}{z^{2}}$ to this order, and gives the boundary metric as [83, 87]

$$
d s^{2}(0)=-\frac{\omega^{2} d \tau^{2}}{\ell^{2}}+\frac{\alpha^{2} \omega^{2} d \xi^{2}}{G F^{4}}+\frac{\alpha^{2} \omega^{2} G d \varphi^{2}}{F^{2} K^{2}} .
$$

The expectation value of the energy momentum of the $C F T_{3}$ is calculated by the following relation 83, 87, 134]

$$
<\mathcal{T}_{a b}>=\lim _{z \longrightarrow 0} \frac{1}{\ell z} \mathcal{T}_{a b}=\frac{3}{2} \rho_{E} U_{a} U_{b}+\frac{\rho_{E}}{2} \ell^{2} \gamma_{a b}^{0}+\pi_{a b}
$$

with $U=\omega^{-1} \partial \tau$. The energy density is

$$
\rho_{E}=\frac{\left(m+2 e^{2} A \xi\right)}{8 \pi \ell^{2} \alpha^{3} \omega^{3}} F^{3}(\xi)\left(2-3 A^{2} \ell^{2} G(\xi)\right)
$$

yielding the mass

$$
M=\int \rho_{E} \ell^{3} \sqrt{-\gamma^{0}} d x d \varphi=\frac{m\left(1-A^{2} \ell^{2}-A^{4} e^{2} \ell^{2}\right)}{K \alpha} .
$$

The variation of the boundary metric with respect to the parameters results to

$$
\delta \gamma^{a b}=\frac{\partial \gamma^{a b}}{\partial K} \delta K+\frac{\partial \gamma^{a b}}{\partial A} \delta A+\frac{\partial \gamma^{a b}}{\partial m} \delta m+\frac{\partial \gamma^{a b}}{\partial e} \delta e,
$$

considering $\ell$ and $\mu$ as constant parameters, one can calculate

$$
\delta I=\int_{\partial M} \sqrt{-\gamma} \tau_{a b} \delta \gamma^{a b} d^{3} x
$$

Imposing that the variation vanishes, the parameter $\alpha$ is obtained as 87

$$
\alpha=\sqrt{\left(1+A^{2} e^{2}\right)\left(1-A^{2} \ell^{2}-A^{4} \ell^{2} e^{2}\right)} .
$$

\section{B: thermodynamic mass}


Setting $m=e=0$ in Eq. (1), we can eliminate the conical singularity [93]. Using the mentioned adjustment, one finds

$$
d s^{2}=\frac{1}{\Omega^{2}}\left[\tilde{f} d t^{2}-\frac{d r^{2}}{\tilde{f}}-r^{2} d \theta^{2}-r^{2} \sin ^{2} \theta d \phi^{2}\right],
$$

where

$$
\tilde{f}=1+\frac{r^{2}}{\ell^{2}}\left(1-A^{2} \ell^{2}\right)
$$

This spacetime no longer has a conical singularity and is locally pure AdS at $r=-\frac{1}{A \cos \theta}($ not $r \rightarrow \infty)$. To transform Rindler coordinate to global AdS coordinates $\{R, \Theta\}$, one takes [83, 87, 98]

$$
R \sin \Theta=\frac{r \sin \theta}{\Omega}, \quad \& \quad R=\ell \sqrt{\frac{\tilde{f}}{\Omega^{2}\left(1-A^{2} \ell^{2}\right)}-1},
$$

which results to the metric of AdS space in global coordinates:

$$
d s^{2}=\left(1+\frac{R^{2}}{\ell^{2}}\right) d t^{2}-\frac{d R^{2}}{1+\frac{R^{2}}{\ell^{2}}}-R^{2}\left(d \Theta^{2}+\sin ^{2} \Theta d \phi^{2}\right) .
$$

[1] J. M. Bardeen, B. Carter, and S. W. Hawking, Commun. Math. Phys. 31, 161 (1973).

[2] S. W. Hawking, Commun. Math. Phys. 43, 199 (1975).

[3] J. D. Bekenstein, Phys. Rev. D 7, 2333 (1973).

[4] J. D. Bekenstein, Phys. Rev. D 9, 3292 (1974).

[5] J. Maldacena, Adv. Theor. Math. Phys. 2, 231 (1998).

[6] E. Witten, Adv. Theor. Math. Phys. 2, 253 (1998).

[7] S. S. Gubser, I. R. Klebanov, and A. M. Polyakov, Phys. Lett. B 428, 105 (1998).

[8] O. Aharony, S. S. Gubser, J. Maldacena, H. Ooguri, and Y. Oz, Phys. Rept. 323, 183 (2000).

[9] D. Bazeia, L. Losano, G. J. Olmo, and D. Rubiera-Garcia, Phys. Rev. D 90, 044011 (2014).

[10] S. W. Hawking, and D. N. Page, Commun. Math. Phys. 87, 577 (1983).

[11] M. Cvetic, and S. S. Gubser, JHEP 04, 024 (1999).

[12] M. Cvetic, and S. S. Gubser, JHEP 07, 010 (1999).

[13] A. Chamblin, R. Emparan, C. Johnson, and R. Myers, Phys. Rev. D 60, 064018 (1999).

[14] A. Chamblin, R. Emparan, C. Johnson, and R. Myers, Phys. Rev. D 60, 104026 (1999).

[15] D. Kubiznak, and R. B. Mann, JHEP 07, 033 (2012).

[16] S. Gunasekaran, D. Kubiznak, and R. B. Mann, JHEP 11, 110 (2012).

[17] R. G. Cai, L. M. Cao, L. Li, and R. Q. Yang, JHEP 09, 005 (2013).

[18] J. X. Mo, and W. B. Liu, Phys. Rev. D 89, 084057 (2014).

[19] M. S. Ma, L. C. Zhang, H. H. Zhao, and R. Zhao, Adv. High Energy Phys. 2015, 134815 (2015).

[20] Y. G. Miao, and Z. M. Xu, Phys. Rev. D 98, 084051 (2018).

[21] R. Zhao, H. Zhao, M. S. Ma, and L. C. Zhang, Eur. Phys. J. C 73, 2645 (2013).

[22] M. H. Dehghani, S. Kamrani, and A. Sheykhi, Phys. Rev. D 90, 104020 (2014).

[23] J. X. Mo, and W. B. Liu, Eur. Phys. J. C 74, 2836 (2014).

[24] S. H. Hendi, S. Panahiyan, and B. Eslam Panah, Prog. Theor. Exp. Phys. 2015, $103 E 01$ (2015).

[25] A. Haldar, and R. Biswas, Gen. Relativ. Gravit. 50, 69 (2018).

[26] B. R. Majhi, and D. Roychowdhury, Class. Quantum Gravit. 29, 245012 (2012).

[27] J. X. Mo, Astrophys. Space Sci. 356, 319 (2015).

[28] Kh. Jafarzade, and J. Sadeghi, Int. J. Mod. Phys. D 26, 1750138 (2017).

[29] M. S. Ma, and R. H. Wang, Phys. Rev. D 96, 024052 (2017).

[30] Y. Z. Du, R. Zhao, and L. C. Zhang, arXiv:1909.09968.

[31] B. Mirza, and Z. Sherkatghanad, Phys. Rev. D 90, 084006 (2014).

[32] J. Xu, L. M. Cao, and Y. P. Hu, Phys. Rev. D 91, 124033 (2015).

[33] S. H. Hendi, B. Eslam Panah, and S. Panahiyan, Class. Quantum Gravit. 33, 235007 (2016).

[34] S. Fernando, Phys. Rev. D 94, 124049 (2016).

[35] S. H. Hendi, S. Panahiyan, B. Eslam Panah, and M. Momennia, Ann. Phys. 528, 819 (2016).

[36] S. H. Hendi, R. B. Mann, S. Panahiyan, and B. Eslam Panah, Phys. Rev. D 95, 021501(R) (2017). 
[37] D. C. Zou, R. Yue, and M. Zhang, Eur. Phys. J. C 77, 256 (2017).

[38] D. C. Zou, Y. Liu, and R. Yue, Eur. Phys. J. C 77, 365 (2017).

[39] S. Upadhyay, B. Pourhassan, and H. Farahani, Phys. Rev. D 95, 106014 (2017).

[40] M. Chabab, H. El Moumni, S. Iraoui, and K. Masmar, Eur. Phys. J. C 79, 342 (2019).

[41] J. X. Mo, G. Q. Li, and Y. C. Wu, JCAP 04, 045 (2016).

[42] A. Ovgün, Adv. High Energy Phys. 2018, 8153721 (2018).

[43] S. H. Hendi, S. Panahiyan, B. Eslam Panah, M. Faizal, and M. Momennia, Phys. Rev. D 94, 024028 (2016).

[44] Z. W. Feng, and S. Z. Yang, Phys. Lett. B 772, 737 (2017).

[45] S. H. Hendi, B. Eslam Panah, and S. Panahiyan, Phys. Lett. B 769, 191 (2017).

[46] Y. S. Myung, Y. W. Kim, and Y. J. Park, Phys. Rev. D 78, 084002 (2008).

[47] S. H. Hendi, and M. H. Vahidinia, Phys. Rev. D 88, 084045 (2013).

[48] S. H. Hendi, G. Q. Li, J. X. Mo, S. Panahiyan, and B. Eslam Panah, Eur. Phys. J. C 76, 571 (2016).

[49] J. X. Mo, G. Q. Li, and X. B. Xu, Phys. Rev. D 93, 084041 (2016).

[50] H. F. Li, H. H. Zhao, L. C. Zhang, and R. Zhao, Eur. Phys. J. C 77, 295 (2017).

[51] M. Zhang, D. C. Zou, and R. H. Yue, Adv. High Energy Phys. 2017, 3819246 (2017).

[52] Z. Dayyani, A. Sheykhi, M. H. Dehghani, and S. Hajkhalili, Eur. Phys. J. C 78, 152 (2018).

[53] P. Wang, H. Wu, and H. Yang, JHEP 07, 002 (2019).

[54] P. Wang, H. Wu, and H. Yang, JCAP 04, 052 (2019).

[55] Y. M. Xu, H. M. Wang, Y. X. Liu, and S. W. Wei, Phys. Rev. D 100, 104044 (2019).

[56] H. Li, Y. Chen, and S. J. Zhang, Nucl. Phys. B 954, 114975 (2020).

[57] S. Grunau, and H. Neumann, Class. Quantum Gravit. 32, 175004 (2015).

[58] B. P. Dolan, Class. Quantum Gravit. 31, 165011 (2014).

[59] S. H. Hendi, and S. Panahiyan, Phys. Rev. D 90, 124008 (2014).

[60] B. Eslam Panah, Phys. Lett. B 787, 45 (2018).

[61] B. Eslam Panah, S. H. Hendi, S. Panahiyan, and M. Hassaine, Phys. Rev. D 98, 084006 (2018).

[62] F. Weinhold, J. Chem. Phys. 63, 2479 (1975).

[63] F. Weinhold, J. Chem. Phys. 63, 2484 (1975).

[64] G. Ruppeiner, Phys. Rev. A 20, 1608 (1979).

[65] G. Ruppeiner, Rev. Mod. Phys. 67, 605 (1995).

[66] P. Salamon, J. Nulton, and E. Ihrig, J. Chem. Phys. 80, 436 (1984).

[67] H. Quevedo, J. Math. Phys. 48, 013506 (2007).

[68] H. Quevedo, and A. Sanchez, JHEP 09, 034 (2008).

[69] H. Quevedo and D. Tapias, J. Math. Chem. 52, 141 (2014).

[70] S. A. H. Mansoori, and B. Mirza, Eur. Phys. J. C 74, 2681 (2014).

[71] S. A. H. Mansoori, B. Mirza, and M. Fazel, JHEP 04, 115 (2015).

[72] S. H. Hendi, S. Panahiyan, B. Eslam Panah, and M. Momennia, Eur. Phys. J. C 75, 507 (2015).

[73] S. H. Hendi, S. Panahiyan, and B. Eslam Panah, Adv. High Energy Phys. 2015, 743086 (2015).

[74] S. H. Hendi, A. Sheykhi, S. Panahiyan, and B. Eslam Panah, Phys. Rev. D 92, 064028 (2015).

[75] S. H. Hendi, B. Eslam Panah, and S. Panahiyan, JHEP 11, 157 (2015).

[76] S. H. Hendi, S. Panahiyan, and B. Eslam Panah, JHEP 01, 129 (2016).

[77] R. Banerjee, B. R. Majhi, and S. Samanta, Phys. Lett. B 767, 25 (2017).

[78] K. Bhattacharya, and B. R. Majhi, Phys. Rev. D 95, 104024 (2017).

[79] V. Pineda, H. Quevedo, M. N. Quevedo, A. Sanchez, and E. Valdes, Int. J. Geom. Meth. Mod. Phys. 11, 1950168 (2019).

[80] M. Appels, R. Gregory, and D. Kubiznak, Phys. Rev. Lett. 117, 131303 (2016).

[81] R. Gregory, J. Phys. Conf. Ser. 942, 012002 (2017).

[82] M. Astorino, Phys. Rev. D 95, 064007 (2017).

[83] A. Anabalon, M. Appels, R. Gregory, D. Kubiznak, R. B. Mann, and A. Ovgün, Phys. Rev. D 98, 104038 (2018).

[84] M. Appels, Thermodynamics of Accelerating Black Holes, Ph.D. thesis, Durham University (2018).

[85] J. Zhang, Y. Li, and H. Yu, Eur. Phys. J. C 78, 645 (2018).

[86] J. Zhang, Y. Li, and H. Yu, JHEP 02, 144 (2019).

[87] A. Anabalon, F. Gray, R. Gregory, D. Kubiznak, and R. B. Mann, JHEP 04, 096 (2019).

[88] N. Abbasvandi, W. Cong, D. Kubiznak, and R. B. Mann, Class. Quantum Gravit. 36, 104001 (2019).

[89] N. Abbasvandi, W. Ahmed, W. Cong, D. Kubiznak, and R. B. Mann, Phys. Rev. D 100, 064027 (2019).

[90] S. Gregory, and A. Scoins, Phys. Lett. B 796, 191 (2019).

[91] B. Eslam Panah, and Kh. Jafarzade, arXiv:1906.09478.

[92] W. Ahmed, H. Z. Chen, E. Gesteau, R. Gregory, and A. Scoins, Class. Quantum Gravit. 36214001 (2019).

[93] M. Appels, R. Gregory, and D. Kubiznak, JHEP 05, 116 (2017).

[94] W. Kinnersley, and M. Walker, Phys. Rev. D 2, 1359 (1970).

[95] J. F. Plebanski, and M. Demianski, Ann. Phys. 98, 98 (1976).

[96] O. J. C. Dias, and J. P. S. Lemos, Phys. Rev. D 67, 064001 (2003).

[97] J. B. Griffiths, and J. Podolsky, Int. J. Mod. Phys. D 15, 335 (2006).

[98] J. Podolsky, Czech. J. Phys. 52, 1 (2002).

[99] M. Zhang, and J. Jiang, Phys. Rev. D 103, 025005 (2021).

[100] A. Ashtekar, and T. Dray, Comm. Math. Phys. 79, 581 (1981). 
[101] J. Podolsky, M. Ortaggio, and P. Krtouss, Phys. Rev. D 68, 124004 (2003).

[102] S. W. Hawking, G. T. Horowitz, and S. F. Ross, Phys. Rev. D 51, 4302 (1995).

[103] R. Emparan, and H. S. Reall, Phys. Rev. Lett. 88, 101101 (2002).

[104] F. Dowker, J. P. Gauntlett, D. A. Kastor, and J. H. Traschen, Phys. Rev. D 49, 2909 (1994).

[105] D. M. Eardley, G. T. Horowitz, D. A. Kastor, and J. H. Traschen, Phys. Rev. Lett 75, 3390 (1995).

[106] S. W. Hawking, and S. F. Ross, Phys. Rev. D 56, 6403 (1997).

[107] M. Zhang, and J. Jiang, Phys. Rev. D 101, 104012 (2020).

[108] T. C. Frost, and V. Perlick, Class. Quantum Grav. 38, 085016 (2021).

[109] K, Destounis, R. D. B. Fontana, and F. C. Mena, Phys. Rev. D 102, 044005 (2020).

[110] S. Jiang, and J. Jiang, arXiv:2106.09371.

[111] K. Destounis, R. D. B. Fontana, and F. C. Mena, Phys. Rev. D 102, 104037 (2020).

[112] S. Guha, and S. Chakraborty, Int. J. Mod. Phys. D 29, 5 (2020).

[113] P. Ferrero, J. P. Gauntlett, J. M. Perez Ipina, D. Martelli, and J. Sparks, arXiv:2012.08530.

[114] M. Tavakoli, B. Mirza, and Z. Sherkatghanad, Nucl. Phys. B 943, 114620 (2019).

[115] Y. Huang, and S. Guo, arXiv:2009.09401.

[116] A. Ball, arXiv:2103.07521.

[117] A. Ball, and N. Miller, Class. Quantum Gravit. 38, 145031 (2021).

[118] M. Zhang, and R. B. Mann, Phys. Rev. D 100, 084061 (2019).

[119] A. Belhaj, H. El Moumni, and K. Masmar, Adv. High Energy Phys. 2020, 4092730 (2020).

[120] M. Rostami, J. Sadeghi, S. Miraboutalebi, A. A. Masoudi, and B. Pourhassan, Int. J. Geome. Meth. Mod. Phys. 17, 2050136 (2020).

[121] J. Podolsky, and A. Vratny, Phys. Rev. D 102, 084024 (2020).

[122] A. Ashtekar, and S. Das, Class. Quantum Gravit. 17, L17 (2000).

[123] S. Das, and R. B. Mann, JHEP 08, 033 (2000).

[124] G. W. Gibbons, M. J. Perry, and C. N. Pope, Class. Quantum Gravit. 22, 1503 (2005).

[125] B. P. Dolan, Class. Quantum Gravit. 28, 125020 (2011).

[126] S. H. Hendi, S. Panahiyan, B. Eslam Panah, and M. Jamil, Chin. Phys. C 43, 113106 (2019).

[127] S. H. Hendi, S. Panahiyan, and B. Eslam Panah, Int. J. Mod. Phys. D 25, 1650010 (2016).

[128] H. Liu, and X. Meng, Mod. Phys. Lett. A 37, 1650199 (2016).

[129] J. X. Mo, and W. B. Liu, Phys. Lett. B 727, 336 (2013).

[130] S. W. Wei, Y. X. Liu, and R. B. Mann, Phys. Rev. D 100, 124033 (2019).

[131] V. Balasubramanian, and P. Kraus, Commun. Math. Phys. 208, 413 (1999).

[132] R. Emparan, C. V. Johnson, and R. C. Myers, Phys. Rev. D 60, 104001 (1999).

[133] R. B. Mann, Phys. Rev. D 60, 104047 (1999).

[134] G. Bernardi de Freitas, and H. S. Reall, JHEP 06, 148 (2014). 